\title{
Cahn-Hilliard equations with memory and dynamic boundary conditions
}

\author{
Cecilia Cavaterra \\ Dipartimento di Matematica "F. Enriques" \\ Università degli Studi di Milano \\ 20133 Milano, ITALY \\ cecilia.cavaterra@unimi.it \\ Ciprian G. Gal \\ Department of Mathematics \\ University of Missouri \\ Columbia, MO 65211, USA \\ galc@missouri.edu \\ Maurizio Grasselli \\ Dipartimento di Matematica "F. Brioschi" \\ Politecnico di Milano \\ 20133 Milano, ITALY \\ maurizio.grasselli@polimi.it
}

April 6, 2010

\begin{abstract}
We consider, on a three dimensional bounded domain, a Cahn-Hiliard equation where the velocity of the order parameter $u$ depends on the past history of $\Delta \mu, \mu$ being the chemical potential. This type of equation has been proposed by P. Galenko et al. to model phase separation phenomena in special materials (e.g. glasses). In addition, the usual no-flux boundary condition for $u$ is replaced by a nonlinear dynamic boundary condition which accounts for possible interactions with the boundary. The resulting boundary value problem is subject to suitable initial conditions and is reformulated in the so-called past history space. Existence of a weak solution is proven. Then, in the viscous case (namely when $\mu$ also depends on $u_{t}$ ), we can also prove uniqueness and construct a strongly continuous semigroup acting on a certain phase space. The
\end{abstract}


corresponding dynamical system has a (smooth) global attractor as well as an exponential attractor. In the non-viscous case, we only establish the existence of a trajectory attractor.

\section{Introduction}

Let $\Omega \subset \mathbb{R}^{3}$ be a given bounded domain with a sufficiently smooth boundary $\Gamma$. The CahnHilliard equation (see [1], cf. also [43] and references therein)

$$
\partial_{t} u=\Delta \mu
$$

with

$$
\mu=\alpha \partial_{t} u-\Delta u+f(u),
$$

in $\Omega \times(\tau, \infty), \tau$ being an arbitrary initial time, plays a basic role in Materials Science. In particular, it gives a good description of the so-called spinodal decomposition of (passive) binary mixtures. This phase separation phenomenon takes place, for example, in cooling processes of metallic alloys, glasses or polymer mixtures. Here $u$ is the relative concentration difference of the mixture components, $\mu$ is the so-called chemical potential and $\alpha \geq 0$ is the viscosity coefficient whose presence accounts for some microscopic effects (see, e.g., [42], cf. also [32]). Moreover, $f$ is the derivative of a potential function $F$ which accounts for the presence of different phases. This potential is a logarithmic-type function defined on a bounded interval (say, $(-1,1)$ ), which is often approximated by a double-well polynomial potential, namely, $F(y)=\left(y^{2}-1\right)^{2}$. Such potentials, defined on the whole $\mathbb{R}$, are usually known as "smooth", while the logarithmic-like are named "singular".

Equations (1.1)-(1.2) are usually subject to the so-called no-flux boundary conditions which entail the conservation of mass. In this case (and, also, with other standard boundary conditions), the corresponding initial and boundary value problem has been extensively studied by many authors (see [43] for some references). In particular, Cahn-Hilliard equation has also been analyzed as a dissipative dynamical system (cf. [52]) and there are satisfactory results on global and exponential attractors (see, for instance, [9]) and the convergence of solutions to steady states (see, e.g., [48]).

More recently, some german physicists have modeled phase separation in binary systems when the dynamic interactions of the material with the walls must be taken into account (cf., for instance, $[10,11,36]$ ). Mathematically speaking, this usually leads to additional terms in the free energy and then to a boundary condition characterized by the presence of $\partial_{t} u$. More precisely, such dynamic condition has the form

$$
\partial_{t} u-\Delta_{\Gamma} u+\partial_{\mathbf{n}} u+\beta u+g(u)=0, \quad \text { on } \Gamma \times(\tau, \infty) .
$$

\footnotetext{
${ }^{1} 2000$ Mathematics Subject Classification: 35B40, 35B41, 45K05, 80A22.

Keywords: Cahn-Hilliard equations, dynamic boundary conditions, global attractors, trajectory attractors, memory relaxation.
} 
Here, the constant $\beta$ is positive, $\Delta_{\Gamma}$ denotes the Laplace-Beltrami operator on the surface $\Gamma$, while $g$ is the derivative of a sufficiently smooth boundary potential $G$. In the original derivation, one has $G(s)=c_{1} y^{2}-c_{2}$, where $c_{1}>0$ accounts for a modification of the effective interaction between the components with the walls, while $c_{2} \geq 0$ (or $c_{2}<0$ ) characterizes the possible preferential attraction (or repulsion) of one of the components by the walls. The mathematical analysis of the Cahn-Hilliard equation endowed with (1.3) and no-flux condition for $\mu$ (to preserve mass conservation), that is,

$$
\partial_{\mathbf{n}} \mu=0, \quad \text { on } \Gamma \times(\tau, \infty),
$$

has recently been the subject of a number of papers. For instance, the case of smooth potentials has been analyzed in $[4,39,46,47,51]$ (see also [12, 14, 31] for non-isothermal models). The more difficult case of singular (e.g., logarithmic) potentials has been considered in $[24,41]$ (cf. also [23] for non-isothermal systems).

On the other hand, a modification of the Cahn-Hiliard equation has proposed to account for rapid spinodal decomposition in certain materials like glasses (see, for instance, $[15,16$, 17, 18, 19, 37]). This modification leads to replacing equation (1.1) with

$$
\partial_{t} u=\int_{0}^{\infty} k(s) \Delta \mu(t-s) d s, \quad \text { in } \Omega \times(\tau, \infty),
$$

where $k:(0, \infty) \rightarrow(0, \infty)$ is a (smooth) decreasing and summable relaxation kernel. This means that relaxation effects are described through a distributed delay. In particular, if $k(s)=\frac{1}{\epsilon} e^{-s / \epsilon}$, where $\epsilon>0$ is a given relaxation time, equation (1.5) is equivalent to a purely differential equation of the form

$$
\epsilon \partial_{t t} u+\partial_{t} u-\Delta \mu=0, \quad \text { in } \Omega \times(\tau, \infty) .
$$

Equation (1.6) has received lot of attention in the last years. The viscous three-dimensional case has been firstly analyzed in [20] (see also [33, 34]). It is worth observing that, in this case, the solutions regularize in finite time (as in the classical case $\epsilon=0$ ). The non-viscous case is much more troublesome even in dimension two since there is a lack of regularization effects (a sort of "hyperbolic" behavior). Only recently, the understanding of the case $\alpha=0$ has significantly improved (see $[27,28,29]$ ). The reader is referred to the quoted papers for more references (e.g. the ones devoted to the non-viscous one dimensional case).

Equation (1.5) with a more general memory kernel (which does not allow to write an equivalent PDE) has been firstly studied in [21] in the one dimensional non-viscous case (see also [22] for no-flux boundary conditions). The corresponding dynamical system has been analyzed within the past history setting with particular regard to the stability with respect to the relaxation time. The three-dimensional non-isothermal non-viscous case has been considered in [38] and the existence of a (weak) solution has been proven. In a related paper (see [50]), well-posedness and regularity results have been obtained by taking memory kernels which are singular at 0 . We recall that this assumption, contrary to nonsingular kernels, produces regularization effects. More recently, equation (1.6) with $\alpha>0$ has been 
carefully analyzed in the spirit of [20]. This means that the kernel $k$ has been rescaled with a relaxation time $\epsilon>0$ and the robustness properties of the dynamical system with respect to $\alpha$ and $\epsilon$ have been investigated. In particular, the authors have constructed a family of exponential attractor which is continuous as $(\alpha, \epsilon)$ goes to $(0,0)$, provided that $\epsilon$ is dominated by $\alpha$. Of course, the latter restriction is expected due to the difficulties arising in the relaxed non-viscous case. In other words, the dynamical system (in the history phase space) generated by (1.6) is "close" to the one corresponding to the original Cahn-Hilliard equation whenever the viscosity and the relaxation time are small. A further recent result concerns (1.5) with $\alpha=0$ in dimension two (see [5]). There, using some ideas of [28], existence of a global (smooth) attractor for energy solutions (see [28] for this terminology) is established for small relaxation times.

All the mentioned results on equation (1.5) (or (1.6)) have been obtained for, say, standard boundary conditions (no-flux, Dirichlet-type or periodic) and smooth potentials. Our goal is to study such equation subject to dynamic boundary conditions with a smooth potential. Handling singular potentials appears out of reach for the moment. More precisely, we consider (1.2)-(1.5) with initial condition on the whole past history of $u$ up to the initial time $\tau$,

$$
u(t)_{\mid t \leq \tau}=u_{0}(t)
$$

for some given $u_{0}$.

We point out that, even in the case $\alpha>0$, the presence of a generic memory kernel implies that the dynamical system has some hyperbolic-like features related to the evolution of the past history variable (see, e.g., [30]). This aspect has also been present in [7]. Nonetheless, in that paper Dirichlet-type boundary conditions have been considered. On the other hand, boundary condition (1.3) brings new additional technical difficulties (even at the well-posedness level) since standard techniques based on the use of fractional powers of operators cannot be exploited (see, for instance, [39]). However, by employing refined energy estimates and regularity theory for suitable elliptic boundary value problems (in the spirit of [39]), we can overcome such difficulties and, successfully, prove the existence of (smooth) global attractors and exponential attractors for (1.2)-(1.5) subject to (1.7), in the viscous case. In the much harder non-viscous case we are only able to establish the existence of an energy solution which satisfies a suitable dissipative estimate. This allows us to prove the existence of a trajectory attractor according to [3] (see also [29, 49] for equation (1.6)). The construction of a family of exponential attractors which is robust with respect to $\alpha$ and $\epsilon$ (as in [7]) seems at present rather difficult to carry out. Apparently, this is due to the lack of a certain regularity property of the solution which cannot be recovered in case of dynamic boundary conditions (see Section 3 for details).

The plan of this paper goes as follows. In Section 2, we introduce the functional framework associated with our problem. Then, in Section 3, we state and prove the well-posedness result by establishing the existence of energy solutions within a suitable Galerkin approximation scheme (see Theorem 3.3). In Section 4, we first demonstrate the existence of bounded absorbing sets and of a compact exponentially attracting set for energy solutions whenever $\alpha>0$. Hence, always in the case $\alpha>0$, we deduce the existence of a (smooth) global 
attractor $\mathcal{A}$ (cf. Theorems 4.1 and 4.5). As a consequence, the global attractor contains only (quasi)strong solutions. The finite dimensionality of $\mathcal{A}$ follows from the existence of an exponential attractor which is proven in Section 5 (see Theorem 5.1). Finally, in Section 6 we show the existence of a trajectory attractor and, consequently, of a weak global attractor in the non-viscous case (see Theorem 6.5)

\section{Past history formulation and functional setup}

In order to formulate problem (1.2)-(1.5), (1.7) in a proper functional setting we will follow the well-established past history approach (see [30], cf. also [7, 21]). To this end, we introduce the past history variable

$$
\eta^{t}(s)=\int_{0}^{s}-\Delta \mu(t-y) d y
$$

for any $s>0$ and $t>\tau$. We observe that $\eta$ satisfies the boundary condition:

$$
\eta^{t}(0)=0, \quad \text { in } \Omega \times(\tau, \infty)
$$

If $k$ is smooth enough and vanishing at $\infty$, then an integration by parts in time of the convolution product appearing in equation (1.5) leads to the following formulation of our original problem, where $\nu=-k^{\prime}$.

Problem P. Find $(u, \psi, \eta)$ with $u(t)_{\mid \Gamma}=\psi(t)$ in $\mathbb{R}$ such that

$$
\begin{aligned}
& \partial_{t} u+\int_{0}^{\infty} \nu(s) \eta(s) d s=0, \quad \text { in } \Omega \times(\tau, \infty), \\
& \partial_{t} \psi-\Delta_{\Gamma} \psi+\partial_{\mathbf{n}} u+\beta \psi+g(\psi)=0, \quad \text { on } \Gamma \times(\tau, \infty), \\
& \mu=\alpha \partial_{t} u-\Delta u+f(u), \quad \text { in } \Omega \times(\tau, \infty), \\
& \partial_{t} \eta+\partial_{s} \eta=-\Delta \mu, \quad \text { in } \Omega \times(\tau, \infty),
\end{aligned}
$$

subject to (1.4), (2.2) and satisfying initial conditions

$$
u(\tau)=u_{0}(\tau), \quad \psi(\tau)=\psi_{0}(\tau), \quad \eta^{\tau}=\eta_{0} .
$$

Note that $\eta_{0}$ is defined as follows

$$
\eta_{0}(s)=\int_{0}^{s}-\Delta \mu_{0}(\tau-y) d y, \quad \text { in } \Omega \times \mathbb{R}_{+},
$$

where $\mu_{0}=\alpha \partial_{t} u_{0}-\Delta u_{0}+f\left(u_{0}\right)$, for $t \leq \tau$. Nevertheless, from now on $\eta_{0}$ will be regarded as an independent initial datum. Therefore we will consider a more general problem with respect to the original one. However, the notion of a solution satisfying (2.3)-(2.7) is still too formal at this point. In order to give a rigorous definition of a solution to $\mathbf{P}$ (cf. Definition 3.1 below), we need to introduce some terminology and the functional setting associated with (2.3)-(2.7). 
In the sequel, we denote by $\|\cdot\|$ and $\|\cdot\|_{\Gamma}$ the norms on $L^{2}(\Omega)$ and $L^{2}(\Gamma)$, whereas the inner products in these spaces are denoted by $(\cdot, \cdot)$ and $(\cdot, \cdot)_{\Gamma}$, respectively. Furthermore, the norms on $H^{r}(\Omega)$ and $H^{r}(\Gamma), r>0$, are indicated by $\|\cdot\|_{H^{r}}$ and $\|\cdot\|_{H^{r}(\Gamma)}$, respectively. The symbol $(\cdot, \cdot)_{V^{*}, V}$ stands for a duality pairing between any generic Banach spaces $V$ and its dual $V^{*}$.

Let us define the linear operator $A_{N}:=-\Delta$ acting on $\mathcal{D}\left(A_{N}\right)=\left\{\theta \in H^{2}(\Omega): \partial_{\mathbf{n}} \theta=0\right.$ on $\Gamma$ \} as the realization in $L^{2}(\Omega)$ of the Laplace operator supplemented with Neumann boundary conditions. It is well known that $A_{N}$ generates a bounded analytic semigroup $e^{-A_{N} t}$ on $L^{2}(\Omega)$ and that $A_{N}$ is nonnegative and self-adjoint on $L^{2}(\Omega)$. Moreover, denoting by $\langle\cdot\rangle$ the spatial average over $\Omega$ and setting $H_{(0)}^{r}(\Omega)=\left\{\phi \in H^{r}(\Omega):\langle\phi\rangle=0\right\}, H^{0}=L^{2}$, we know that $A_{N}^{-1}: H_{(0)}^{0}(\Omega) \rightarrow H_{(0)}^{0}(\Omega)$ is well defined. Henceforth, we will always refer to the following norms in $H^{-r}(\Omega):=\left(H^{r}(\Omega)\right)^{*}, r \in \mathbb{N}_{+}$, which are equivalent to the standard ones,

$$
\|u\|_{H^{-r}}^{2}=\left\|A_{N}^{-r / 2}(u-\langle u\rangle)\right\|^{2}+\langle u\rangle^{2} .
$$

The Dirichlet trace map $\operatorname{Tr}_{D}: C^{\infty}(\bar{\Omega}) \rightarrow C^{\infty}(\Gamma)$, defined by $\operatorname{Tr}_{D}(u)=u_{\mid \Gamma}$ extends to a linear continuous operator $\operatorname{Tr}_{D}: H^{r}(\Omega) \rightarrow H^{r-1 / 2}(\Gamma)$, for all $r>1 / 2$, which is onto for $1 / 2<r<3 / 2$. This map also possesses a bounded right inverse $\operatorname{Tr}_{D}^{-1}: H^{r-1 / 2}(\Gamma) \rightarrow H^{r}(\Omega)$ such that $\operatorname{Tr}_{D}\left(\operatorname{Tr}_{D}^{-1} \psi\right)=\psi$, for any $\psi \in H^{r-1 / 2}(\Gamma)$. We can thus introduce the subspaces of $H^{r}(\Omega) \times H^{r}(\Gamma)$,

$$
\mathbb{V}_{r}:=\left\{(u, \psi) \in H^{r}(\Omega) \times H^{r}(\Gamma): \operatorname{Tr}_{D}(u)=\psi\right\},
$$

for every $r>1 / 2$, and note that we have the following dense and compact embeddings $\mathbb{V}_{r_{1}} \subset \mathbb{V}_{r_{2}}$, for any $r_{1}>r_{2}>1 / 2$. We emphasize that $\mathbb{V}_{r}$ is not a product space.

Let us now introduce the spaces for the memory variable $\eta$. For a nonnegative measurable function $\theta$ defined on $\mathbb{R}_{+}$and a real Hilbert space $W$ (with inner product denoted by $(\cdot, \cdot)_{W}$ ), let $L_{\theta}^{2}\left(\mathbb{R}_{+} ; W\right)$ be the Hilbert space of $W$-valued functions on $\mathbb{R}_{+}$, endowed with the following inner product

$$
\left(\phi_{1}, \phi_{2}\right)_{L_{\theta}^{2}\left(\mathbb{R}_{+} ; W\right)}=\int_{0}^{\infty} \theta(s)\left(\phi_{1}(s), \phi_{2}(s)\right)_{W} d s .
$$

Consequently, we set

$$
\mathcal{M}_{\sigma}=L_{\nu}^{2}\left(\mathbb{R}_{+} ; H^{\sigma}(\Omega)\right), \quad \mathcal{M}_{\sigma}^{(0)}=L_{\nu}^{2}\left(\mathbb{R}_{+} ; H_{(0)}^{\sigma}(\Omega)\right)
$$

for $\sigma \in \mathbb{R}$. In the sequel, we will also consider Hilbert spaces of the form $H_{\nu}^{k}\left(\mathbb{R}_{+} ; H^{\sigma}(\Omega)\right)$ for $k \in \mathbb{N}$.

We conclude by observing that solutions to problem $\mathbf{P}$ must also satisfy the following constraints (i.e., mass conservation)

$$
\langle u(t)\rangle=\left\langle u_{0}(\tau)\right\rangle, \quad\left\langle\eta^{t}(s)\right\rangle=0
$$

for $t>\tau$ and $s>0$. These can be easily derived recalling equations (1.5), (1.4) and using (2.1). For this reason we need to introduce closed subsets of Hilbert spaces $\mathcal{H}_{r, \sigma}=\mathbb{V}_{r} \times \mathcal{M}_{\sigma-1}^{(0)}$ 
for $r>1 / 2, \sigma \in \mathbb{R}$, endowed with the scalar product induced by $\mathbb{V}_{r} \times \mathcal{M}_{\sigma-1}^{(0)}$, namely,

$$
\mathcal{H}_{r, \sigma}^{M}=\left\{(u, \psi, \eta) \in \mathbb{V}_{r} \times \mathcal{M}_{\sigma-1}^{(0)}:|\langle u\rangle| \leq M\right\}
$$

for some given $M \geq 0$. Note that $\mathcal{H}_{r, \sigma}^{M}$ are complete metric spaces with respect to the metrics induced by the norms of $\mathcal{H}_{r, \sigma}$. We now have defined all the ingredients we need to introduce a rigorous formulation of $\mathbf{P}$ in the next section.

\section{Variational formulation and well-posedness}

We begin this section by stating all the hypotheses on $\nu, f$ and $g$ we need, even though not all of them must be satisfied at the same time.

\section{Conditions on $\nu$ :}

$$
\begin{aligned}
& \nu \in C^{1}\left(\mathbb{R}_{+}\right) \cap L^{1}\left(\mathbb{R}_{+}\right): \nu(s) \geq 0, \quad \forall s \in \mathbb{R}_{+}, \\
& \nu^{\prime}(s) \leq 0, \quad \forall s \in \mathbb{R}_{+}, \\
& k_{0}=\int_{0}^{\infty} \nu(s) d s>0 \\
& \nu_{0}=\lim _{s \rightarrow 0^{+}} \nu(s)<\infty, \\
& \nu^{\prime}(s)+\lambda \nu(s) \leq 0, \quad \text { for a.a. } s \in \mathbb{R}_{+}
\end{aligned}
$$

for some $\lambda>0$. In the rest of the paper we will take $k_{0}=1$ for the sake of simplicity. Also, we recall that assumption (K5) plays a role in the long term behavior only.

Conditions on $f$ and $g$ :

$$
\begin{aligned}
& f, g \in C^{1}(\mathbb{R}), \\
& f, g \in C^{2}(\mathbb{R}), \\
& \liminf _{|y| \rightarrow+\infty} f^{\prime}(y)>0, \quad \liminf _{|y| \rightarrow+\infty} g^{\prime}(y)>0, \\
& \left|f^{\prime}(y)\right| \leq c_{f}\left(1+|y|^{2}\right), \quad\left|g^{\prime}(y)\right| \leq c_{g}\left(1+|y|^{q}\right), \quad \forall y \in \mathbb{R}, \\
& \left|f^{\prime \prime}(s)\right| \leq c_{f}(1+|s|), \quad\left|g^{\prime \prime}(s)\right| \leq c_{g}\left(1+|s|^{q-1}\right), \quad \forall s \in \mathbb{R},
\end{aligned}
$$

for some positive constants $c_{f}, c_{g}$ and some $q \in[1,+\infty)$. Note that, for instance, doublewell smooth potentials like $F(s)=\left(s^{2}-1\right)^{2}$ are such that $f=F^{\prime}$ satisfies all the above assumptions, while $g$ can be any polynomial of odd degree and positive leading coefficient.

In order to introduce the variational formulation of $\mathbf{P}$ we consider the sesquilinear form $\Upsilon$ in $L^{2}(\Omega) \times L^{2}(\Gamma)$ defined by

$$
\Upsilon(\mathbf{u}, \mathbf{v})=\int_{\Omega} \nabla u \cdot \nabla v d x+\int_{\Gamma} \nabla_{\Gamma} \psi \cdot \nabla_{\Gamma} \xi d S+\beta \int_{\Gamma} \psi \xi d S
$$


for all $\mathbf{u}=(u, \psi), \mathbf{v}=(v, \xi) \in \mathbb{V}_{1}$. Then, $\Upsilon$ (with domain $\left.D(\Upsilon)=\mathbb{V}_{1}\right)$ is densely defined, closed, symmetric and bounded from below in $L^{2}(\Omega) \times L^{2}(\Gamma)$. Thus, by first and second representation theorems for forms (see, e.g., [35, Section VI.2]), one concludes that there exists a unique self-adjoint operator $A_{\Gamma}$ in $L^{2}(\Omega) \times L^{2}(\Gamma)$, bounded from below, associated with the form $\Upsilon$, which satisfies

$$
\Upsilon(\mathbf{u}, \mathbf{v})=\left(\mathbf{u}, A_{\Gamma} \mathbf{v}\right)_{L^{2}(\Omega) \times L^{2}(\Gamma)}=\int_{\Omega} u(-\Delta v) d x+\int_{\Gamma} \psi\left(-\Delta_{\Gamma} \xi+\partial_{\mathbf{n}} v+\beta \xi\right) d S,
$$

for all $\mathbf{u} \in D(\Upsilon), \mathbf{v} \in D\left(A_{\Gamma}\right)$. Moreover, $D\left(A_{\Gamma}\right) \subset D\left(A_{\Gamma}^{1 / 2}\right)=D(\Upsilon)$ is such that

$$
D\left(A_{\Gamma}\right)=\left\{(v, \xi) \in \mathbb{V}_{1}: \Delta v \in L^{2}(\Omega), \quad-\Delta_{\Gamma} \xi+\partial_{\mathbf{n}} v+\beta \xi \in L^{2}(\Gamma)\right\} .
$$

Besides, from (3.1)-(3.2), we deduce that $A_{\Gamma}$ is bounded from $\mathbb{V}_{1}$ into $\mathbb{V}_{1}^{*}$, and according to [13, Section 3], we have the following more explicit characterization of the domain for $A_{\Gamma}$, that is, $D\left(A_{\Gamma}\right)=\mathbb{V}_{2}$. Indeed, the map $\Xi: \mathbf{u} \mapsto A_{\Gamma} \mathbf{u}$, when viewed as a map from $\mathbb{V}_{2}$ into $L^{2}(\Omega) \times L^{2}(\Gamma)$ is an isomorphism and, cf. e.g., [2, Lemma 2.2], there exists a positive constant $C_{*}$, independent of $(u, \psi)$, such that

$$
C_{*}^{-1}\|(u, \psi)\|_{\mathbb{V}_{2}} \leq\|\Xi(\mathbf{u})\|_{L^{2}(\Omega) \times L^{2}(\Gamma)} \leq C_{*}\|(u, \psi)\|_{\mathbb{V}_{2}},
$$

for all $\mathbf{u}=(u, \psi) \in \mathbb{V}_{2}$.

To formulate the evolution equation for $\eta$ we need to define the linear operator $\mathbb{T}=-\partial_{s}$ with domain

$$
D(\mathbb{T})=\left\{\eta \in \mathcal{M}_{-1}^{(0)}: \partial_{s} \eta \in \mathcal{M}_{-1}^{(0)}, \eta(0)=0\right\} .
$$

We recall that $\mathbb{T}$ is the infinitesimal generator of the right translation semigroup on $\mathcal{M}_{-1}$ (see, e.g., [30]).

We are now ready to introduce the rigorous (variational) formulation of problem $\mathbf{P}$.

Definition 3.1 Let $T>\tau$ and set $J=[\tau, T]$. A triplet $(u, \psi, \eta)$ which fulfills

$$
\begin{aligned}
& (u, \psi, \eta) \in L^{\infty}\left(J ; \mathcal{H}_{1,0}\right), \\
& \partial_{t} u \in L^{\infty}\left(J ; H^{-1}(\Omega)\right), \quad \alpha^{1 / 2} \partial_{t} u \in L^{2}(\Omega \times J), \quad \partial_{t} \psi \in L^{2}(\Gamma \times J),
\end{aligned}
$$

is a solution to problem $\boldsymbol{P}$ in $J$ with initial data $\left(u_{0}, \psi_{0}, \eta_{0}\right) \in \mathcal{H}_{1,0}$ if the following identities hold almost everywhere in $J$ :

$$
\begin{aligned}
& \left(\partial_{t} u, v\right)_{H^{-1}, H^{1}}+\int_{0}^{\infty} \nu(s)\left(\eta^{t}(s), v\right)_{H^{-1}, H^{1}} d s=0, \quad \forall v \in H^{1}(\Omega), \\
& \Upsilon(\mathbf{u}, \mathbf{v})+(f(u), v)+(g(\psi), \varphi)_{\Gamma}+\left(\alpha \partial_{t} u, v\right)+\left(\partial_{t} \psi, \varphi\right)_{\Gamma} \\
& =(\mu, v)_{H^{-1}, H^{1}}, \quad \forall \mathbf{v}=(v, \varphi) \in \mathbb{V}_{1} \\
& \left(\partial_{t} \eta-\mathbb{T} \eta, \zeta\right)_{\mathcal{M}_{-1}}=(\mu, \zeta)_{\mathcal{M}_{0}}, \quad \forall \zeta \in \mathcal{M}_{1} .
\end{aligned}
$$

As a consequence of (3.7), there holds

$$
\langle u(t)\rangle=\left\langle u_{0}\right\rangle, \quad \text { for a.a. } t \in J .
$$


Remark 3.2 As far as the interpretation of the initial condition is concerned, note that properties (3.5)-(3.6) imply that $\mathbf{u}=(u, \psi) \in C\left(J ; \mathbb{V}_{1-r}\right)$ for any $r \in(0,1 / 2)$. On the other hand, it can be shown that $\eta \in C\left(J ; \mathcal{M}_{-1}\right)$ (see [30]).

We now state and prove the following global existence result. Note that the notion of solutions we are looking for is far weaker than the usual notion of weak solutions. Solutions of this kind have been called energy solutions in [28].

Theorem 3.3 Let (K1)-(K4) and (N0),(N1),(N2) hold. Then, for any given initial time $\tau \in \mathbb{R}$ and $\left(u_{0}, \psi_{0}, \eta_{0}\right) \in \mathcal{H}_{1,0}$, problem $\boldsymbol{P}$ admits at least one solution $(u, \psi, \eta)$ on the time interval J, according to Definition 3.1. In addition, this solution satisfies the following inequality:

$$
\left\|\left(u(t), \psi(t), \eta^{t}\right)\right\|_{\mathcal{H}_{1,0}}^{2}+\int_{\tau}^{t}\left(\alpha\left\|\partial_{t} u(y)\right\|^{2}+\left\|\partial_{t} \psi(y)\right\|_{\Gamma}^{2}\right) d y \leq Q\left(\left\|\left(u_{0}, \psi_{0}, \eta_{0}\right)\right\|_{\mathcal{H}_{1,0}}^{2}\right),
$$

for $t \geq \tau$, for some positive monotone nondecreasing function $Q$ independent of $t$ and $\alpha$.

Proof. The proof is divided into three steps. We make use of a Faedo-Galerkin approximating scheme (similar to $[12,24]$ ) to project problem $\mathbf{P}$ on a finite dimensional space, where we can locally solve a Cauchy problem for a system of ordinary differential equations $\mathbf{P}_{n}$, having local solutions. Then, we provide some a priori estimates which guarantee that any local solution to problem $\mathbf{P}_{n}$ is actually global on $J$. The a priori boundedness is also used to extract a subsequence which converges weakly to a certain vector-valued function, candidate to be the solution to problem $\mathbf{P}$.

Step 1: The Galerkin approximation. We begin by choosing a smooth orthonormal basis $\left\{v_{j}\right\}, j \in \mathbb{N}_{+}$, in $L^{2}(\Omega)$, which can be achieved, for instance, by taking the set $\left\{v_{j}\right\}$ of the eigenfunctions (normalized in $L^{2}(\Omega)$ ) for the operator $A_{N}$ in $L^{2}(\Omega)$, that is, $A_{N} v_{j}=\varpi_{j} v_{j}$, $j \in \mathbb{N}_{+}$, where $\left\{v_{j}\right\} \subset D\left(A_{N}\right)$ and $\varpi_{j}$ is the eigenvalue corresponding to $v_{j}$. In particular, it is well-known that $\varpi_{1}=0$ is simple with constant eigenfunction $v_{1}=|\Omega|^{-1 / 2}$, while all the other eigenvalues $\varpi_{j}, j \geq 2$, are positive and can be increasingly ordered and counted according to their multiplicities in order to form a real divergent sequence. Next, we take a smooth sequence $\left\{z_{j}\right\} \subset H_{\nu}^{1}\left(\mathbb{R}_{+} ; H_{(0)}^{1}(\Omega)\right) \cap D(\mathbb{T})$ forming an orthonormal basis of $\mathcal{M}_{-1}^{(0)}$. For $n \in \mathbb{N}$, we consider the projections $\mathcal{O}_{n}$ and $\mathcal{Q}_{n}$, given by

$$
\begin{aligned}
& \mathcal{O}_{n}: H^{1}(\Omega) \rightarrow V^{n}=\operatorname{span}\left\{v_{1}, v_{2}, \ldots, v_{n}\right\}, \\
& \mathcal{Q}_{n}: \mathcal{M}_{-1}^{(0)} \rightarrow \mathcal{M}^{n}=\operatorname{span}\left\{z_{1}, z_{2}, \ldots, z_{n}\right\}
\end{aligned}
$$

and set

$$
V^{\infty}=\cup_{n=1}^{\infty} V^{n}, \quad \mathcal{M}^{\infty}=\cup_{n=1}^{\infty} \mathcal{M}^{n} .
$$

Clearly, $V^{\infty}$ is a dense subspace of $H^{1}(\Omega)$ and thus, of $L^{2}(\Gamma)$, since $D\left(A_{N}\right)$ is densely contained in $L^{2}(\Gamma)$. Moreover, $\mathcal{M}^{\infty}$ is a dense subspace of $\mathcal{M}_{-1}^{(0)}$, respectively. Furthermore, 
for each $v_{n} \in V^{n} \subset C(\bar{\Omega})$, we can define elements of the form $\mathbf{v}_{n}=\left(v_{n}, w_{n}\right)$, with $w_{n}=$ $\operatorname{Tr}_{D}\left(v_{n}\right)$ and note that

$$
\overline{\mathbb{V}}^{\infty}:=\cup_{n=1}^{\infty} \operatorname{span}\left\{\mathbf{v}_{1}, \mathbf{v}_{2}, \ldots, \mathbf{v}_{n}\right\}
$$

is dense in $\mathbb{V}_{1}$ (see, e.g., [24]). It is convenient to introduce a further projector on the trace component

$$
\widetilde{\mathcal{O}}_{n}: H^{1}(\Omega) \rightarrow \widetilde{V}^{n}=\operatorname{span}\left\{w_{1}, w_{2}, \ldots, w_{n}\right\} .
$$

For each given $n \in \mathbf{N}$ our approximating problem is

Problem $\mathbf{P}_{n}$. Find $t_{n} \in(\tau, T]$ and $a_{j}:=a_{j}^{n} \in C^{1}\left(\left[\tau, t_{n}\right] ; \mathbb{R}^{n}\right), j=1,2,3$, such that

$$
\begin{aligned}
u_{n}(t) & =\sum_{j=1}^{n} a_{1 j}(t) v_{j}, \quad \psi_{n}(t)=\sum_{j=1}^{n} a_{1 j}(t) w_{j}, \\
\eta_{n}^{t}(s) & =\sum_{j=1}^{n} a_{2 j}(t) z_{j}(s), \quad \mu_{n}(t)=\sum_{j=1}^{n} a_{3 j}(t) v_{j},
\end{aligned}
$$

solve the following approximating problem

$$
\begin{aligned}
& \left(\partial_{t} u_{n}, v\right)+\int_{0}^{\infty} \nu(s)\left(\eta_{n}(s), v\right) d s=0 \\
& \Upsilon\left(\mathbf{u}_{n}, \mathbf{v}\right)+\left(f\left(u_{n}\right), v\right)+\left(g\left(\psi_{n}\right), w\right)_{\Gamma}+\left(\alpha \partial_{t} u_{n}, v\right)+\left(\partial_{t} \psi_{n}, w\right)_{\Gamma}=\left(\mu_{n}, v\right) \\
& \left(\partial_{t} \eta_{n}-\mathbb{T} \eta_{n}, \zeta\right)_{\mathcal{M}_{-1}}=\left(\mu_{n}, \zeta\right)_{\mathcal{M}_{0}},
\end{aligned}
$$

for every $t \in\left(\tau, t_{n}\right)$ and every $\mathbf{v}=(v, w) \in X_{n}$ and $\zeta \in \mathcal{M}^{n}$, respectively, and with

$$
u_{n}(\tau)=\mathcal{O}_{n} u_{0}, \quad \psi_{n}(\tau)=\widetilde{\mathcal{O}}_{n} u_{0}, \quad \eta_{n}^{\tau}=\mathcal{Q}_{n} \eta_{0}
$$

Let us write (3.14)-(3.17) as a Cauchy problem for a nonlinear system of ordinary differential equations, choosing $\bar{v}=\left(v_{i}, w_{i}\right)$ in $(3.14)-(3.15)$ and $\zeta=z_{i}$ in $(3.16)$, for $i=1, \ldots, n$. To this end, we define the $n \times n$ matrices $\mathbb{B}_{\Gamma}=\left(l_{i j}\right), \mathbb{B}=\left(b_{i j}\right), \mathbb{D}=\left(d_{i j}\right)$ and $\overline{\mathbb{D}}=\left(\bar{d}_{i j}\right)$ given by

$$
l_{i j}=\left(w_{j}, w_{i}\right)_{\Gamma}, \quad b_{i j}=\left(z_{j}, v_{i}\right)_{\mathcal{M}_{-1}}, \quad d_{i j}=\left(\mathbb{T} z_{j}, z_{i}\right)_{\mathcal{M}_{-1}}, \quad \bar{d}_{i j}=\varpi_{j}\left(v_{j}, z_{i}\right)_{\mathcal{M}_{-1}} .
$$

Observe that $\mathbb{B}_{\Gamma}$ is positive semidefinite by construction. After performing direct computations in (3.14)-(3.16) and taking (3.18) into account, we can rewrite $\mathbf{P}_{n}$ into a Cauchy problem for the following system

$$
\begin{aligned}
& \mathbf{a}_{1}^{\prime}=-\mathbb{B} \mathbf{a}_{2}, \\
& \mathbf{a}_{2}^{\prime}=\mathbb{D} \mathbf{a}_{2}+\overline{\mathbb{D}} \mathbf{a}_{3}, \\
& \mathbf{a}_{3}=\mathbb{C}\left(\mathbf{a}_{1}\right)+\left(\alpha \mathbb{I}+\mathbb{B}_{\Gamma}\right) \mathbf{a}_{1}^{\prime},
\end{aligned}
$$


where $\mathbb{I}$ is the $n \times n$ identity matrix and $\mathbb{C}\left(\mathbf{a}_{1}\right)=\left(c_{i}\left(\mathbf{a}_{1}\right)\right): \mathbb{R}^{n} \rightarrow \mathbb{R}^{n}$ is given by

$$
\begin{aligned}
c_{i}\left(\mathbf{a}_{1}\right) & =\sum_{j=1}^{n} a_{1 j} \Upsilon\left(\mathbf{v}_{j}, \mathbf{v}_{i}\right) \\
& +\left(f\left(\sum_{j=1}^{n} a_{1 j} v_{j}\right), v_{i}\right)+\left(g\left(\sum_{j=1}^{n} a_{1 j} w_{j}\right), w_{i}\right)_{\Gamma}, \quad i=1, \ldots, n .
\end{aligned}
$$

It is now easy to deduce from (3.19)-(3.21) a system of $2 n$ nonlinear ordinary differential equations in normal form for the vector-valued functions $\mathbf{a}_{j}=\left(a_{j 1}, \ldots, a_{j n}\right), j=1,2$, that is,

$$
\left(\begin{array}{l}
\mathbf{a}_{1}^{\prime} \\
\mathbf{a}_{2}^{\prime}
\end{array}\right)=\mathbb{U}_{n}\left(\begin{array}{l}
\mathbf{a}_{1} \\
\mathbf{a}_{2}
\end{array}\right),
$$

where $\mathbb{U}_{n}: \mathbb{R}^{2 n} \rightarrow \mathbb{R}^{2 n}$ can be computed explicitly. On account of (N0), we can thus apply the Cauchy-Lipschitz theorem and find a unique maximal solution $\left(u_{n}, \psi_{n}, \eta_{n}\right) \in C^{1}\left(\left[\tau, t_{n}\right] ; \mathcal{H}_{2,2}\right)$ to $\mathbf{P}_{n}$, for some $t_{n} \in(\tau, T)$.

Step 2: A priori estimates. We will now deduce some a priori estimates on the approximating solutions $\left(u_{n}, \psi_{n}, \eta_{n}\right)$. Such estimates, in particular, ensure that the solutions obtained are defined on the whole interval $J=[\tau, T]$, for every $n \in \mathbb{N}$. Throughout this proof, $c$ will denote a positive generic constant, depending at most on the physical parameters of the problem, but independent of $n$ and $\alpha$. Such a constant may vary even within the same line. Further dependencies of the constants will be pointed out if needed.

We first take $v=\mu_{n}(t) \in V_{n}$ and $\mathbf{v}=\left(\partial_{t} u_{n}(t), \partial_{t} \psi_{n}(t)\right) \in X_{n}$ in (3.14) and (3.15), respectively. Adding these relations together, we deduce, for all $t \in\left[\tau, t_{n}\right)$,

$$
\begin{aligned}
& \frac{1}{2} \frac{d}{d t}\left[\left\|\nabla u_{n}(t)\right\|^{2}+\left\|\nabla_{\Gamma} \psi_{n}(t)\right\|_{\Gamma}^{2}+\beta\left\|\psi_{n}(t)\right\|_{\Gamma}^{2}+2\left(G\left(\psi_{n}(t)\right), 1\right)_{\Gamma}+2\left(F\left(u_{n}(t)\right), 1\right)\right] \\
& +\alpha\left\|\partial_{t} u_{n}(t)\right\|^{2}+\left\|\partial_{t} \psi_{n}(t)\right\|_{\Gamma}^{2}+\int_{0}^{\infty} \nu(s)\left(\eta_{n}^{t}(s), \mu_{n}(t)\right) d s=0 .
\end{aligned}
$$

Next, recalling that $\left\langle\eta_{n}^{t}(s)\right\rangle=\left\langle\mathbb{T} \eta_{n}^{t}(s)\right\rangle=0$, take $\zeta=\eta_{n}^{t}(s)$ in (3.16). This yields

$$
\frac{1}{2} \frac{d}{d t}\left\|\eta_{n}^{t}\right\|_{\mathcal{M}_{-1}}^{2}-\int_{0}^{\infty} \nu^{\prime}(s)\left\|\eta_{n}^{t}(s)\right\|_{H^{-1}}^{2} d s=\left(\mu_{n}, \eta_{n}^{t}(s)\right)_{\mathcal{M}_{0}}, \quad \forall t \in\left[\tau, t_{n}\right)
$$

Set now, for all $t \in\left[\tau, t_{n}\right)$,

$$
\begin{aligned}
E_{n}(t) & :=\left\|\nabla u_{n}(t)\right\|^{2}+\left\|\nabla_{\Gamma} \psi_{n}(t)\right\|_{\Gamma}^{2}+\beta\left\|\psi_{n}(t)\right\|_{\Gamma}^{2}+\left\|\eta_{n}^{t}\right\|_{\mathcal{M}_{-1}}^{2} \\
& +2\left(F\left(u_{n}(t)\right), 1\right)+2\left(G\left(\psi_{n}(t)\right), 1\right)_{\Gamma}+\widetilde{C} .
\end{aligned}
$$

Here the constant $\widetilde{C}>0$ is taken large enough in order to ensure that $E_{n}(t)$ is nonnegative (note that, due to assumptions (N1), $F$ and $G$ are both bounded from below, independently 
of $n)$. Adding now (3.24)-(3.25), we obtain

$$
\frac{d}{d t} E_{n}(t)+2 \alpha\left\|\partial_{t} u_{n}(t)\right\|^{2}+2\left\|\partial_{t} \psi_{n}(t)\right\|_{\Gamma}^{2}-2 \int_{0}^{\infty} \nu^{\prime}(s)\left\|\eta_{n}^{t}(s)\right\|_{H^{-1}}^{2} d s=0,
$$

for all $t \in\left[\tau, t_{n}\right]$. Then integrating (3.26) with respect to time, on account of assumption (K2), we get

$$
E_{n}(t)+\int_{\tau}^{t}\left[2 \alpha\left\|\partial_{t} u_{n}(y)\right\|^{2}+2\left\|\partial_{t} \psi_{n}(y)\right\|_{\Gamma}^{2}\right] d y \leq E_{n}(\tau), \quad \forall t \in\left[\tau, t_{n}\right) .
$$

Observe that, in light of (N1)-(N2), there exists a positive constant $c$ independent of the initial data such that

$$
c\left\|\left(u_{n}(t), \psi_{n}(t), \eta_{n}^{t}\right)\right\|_{\mathcal{H}_{1,0}}^{2} \leq E_{n}(t) \leq Q\left(\left\|\left(u_{n}(t), \psi_{n}(t), \eta_{n}^{t}\right)\right\|_{\mathcal{H}_{1,0}}\right), \quad \forall t \in\left[\tau, t_{n}\right),
$$

for some monotone non-decreasing function $Q$ independent of $n$. In particular, the uniform bound (3.27) implies that the local solution to $\mathbf{P}_{n}$ can be extended up to time $T$, that is, $t_{n}=T$, for every $n$.

By (3.27)-(3.28), we learn that the sequence $\left(u_{n}, \psi_{n}, \eta_{n}\right)$ is uniformly bounded in the space $\mathcal{H}_{1,0}$ by a constant depending only on the size of initial data and of the nonlinearities $f, g$. Integrating (3.26) over $J$, we get a further uniform bound for $\partial_{t} u_{n}$ and $\partial_{t} \psi_{n}$ in $L^{2}(\Omega \times J)$ and $L^{2}(\Gamma \times J)$, respectively. Also we get a control of $\eta_{n}$ in $L^{2}\left(J ; L_{\nu^{\prime}}^{2}\left(\mathbb{R}_{+} ; H^{-1}(\Omega)\right)\right)$. Summing up, we have

$$
\begin{aligned}
& \left\|\left(u_{n}, \psi_{n}\right)\right\|_{L^{\infty}\left(J ; \mathbb{V}_{1}\right)} \leq c, \quad \sqrt{\alpha}\left\|\partial_{t} u_{n}\right\|_{L^{2}(\Omega \times J)} \leq c, \\
& \left\|\eta_{n}\right\|_{L^{\infty}\left(J ; \mathcal{M}_{-1}\right)} \leq c, \quad\left\|\eta_{n}\right\|_{L^{2}\left(J ; L_{\nu^{\prime}}^{2}\left(\mathbb{R}_{+} ; H^{-1}(\Omega)\right)\right)} \leq c, \quad\left\|\partial_{t} \psi_{n}\right\|_{L^{2}(\Gamma \times J)} \leq c .
\end{aligned}
$$

Actually we can prove some more regularity properties for $\left(u_{n}, \psi_{n}, \eta_{n}\right)$. To this end, take $v=A_{N}^{-1} \partial_{t} u_{n}(t)$ and observe that $\left\langle\partial_{t} u_{n}(t)\right\rangle=0$. Exploiting the basic Hölder and Young inequalities, it is not difficult to realize, on account of (K3), that

$$
\left\|\partial_{t} u_{n}(t)\right\|_{H^{-1}} \leq\left\|\eta_{n}^{t}\right\|_{\mathcal{M}_{-1}}, \quad \forall t \in J .
$$

Thus, (3.30) and (3.31) yield

$$
\left\|\partial_{t} u_{n}\right\|_{L^{\infty}\left(J ; H^{-1}(\Omega)\right)} \leq c .
$$

Furthermore, thanks to the first bound of (3.29), we can easily control the nonlinear terms in (3.15). In particular, on account of (N2) and the embeddings $H^{1}(\Omega) \hookrightarrow L^{6}(\Omega), H^{1}(\Gamma) \hookrightarrow$ $L^{p}(\Gamma)$, for all $p \in[1, \infty)$, we have

$$
\left\|f\left(u_{n}\right)\right\|_{L^{2}(\Omega \times J)} \leq c, \quad\left\|g\left(\psi_{n}\right)\right\|_{L^{q+1}(\Gamma \times J)} \leq c .
$$

Finally, by comparison in (3.15), the fact that the operator $A_{\Gamma}$ is bounded from $\mathbb{V}_{1}$ into $\mathbb{V}_{1}^{*}$ (in particular, $\left.\left\|A_{\Gamma} u_{n}\right\|_{L^{2}\left(J ; \mathbb{V}_{1}^{*}\right)} \leq c\right)$ combined with (3.33) also provides the following uniform bounds for $\mu_{n}$

$$
\left\langle\mu_{n}\right\rangle \leq c, \quad\left\|\mu_{n}\right\|_{L^{2}\left(J ; H^{-1}(\Omega)\right)} \leq c .
$$


Step 3: Passage to limit. On account of the above uniform inequalities, we can argue, up to subsequences, that

$$
\begin{aligned}
& \left(u_{n}, \psi_{n}\right) \rightarrow(u, \psi) \quad w^{*}-L^{\infty}\left(J ; \mathbb{V}_{1}\right), \quad \partial_{t} \psi_{n} \rightarrow \partial_{t} \psi \quad w-L^{2}\left(J ; L^{2}(\Gamma)\right), \\
& \partial_{t} u_{n} \rightarrow \partial_{t} u \quad w^{*}-L^{\infty}\left(J ; H^{-1}(\Omega)\right), \quad \sqrt{\alpha} \partial_{t} u_{n} \rightarrow \sqrt{\alpha} \partial_{t} u \quad w-L^{2}(\Omega \times J), \\
& \eta_{n} \rightarrow \eta \quad w^{*}-L^{\infty}\left(J ; \mathcal{M}_{-1}\right), \quad \mu_{n} \rightarrow \mu \quad w-L^{2}\left(J ; H^{-1}(\Omega)\right), \\
& \eta_{n} \rightarrow \eta \quad w-L^{2}\left(J ; L_{\nu^{\prime}}^{2}\left(\mathbb{R}_{+} ; H^{-1}(\Omega)\right)\right) .
\end{aligned}
$$

Due to (3.35)-(3.37) and classical compactness theorems, we also have

$$
\begin{aligned}
& u_{n} \rightarrow u \quad \text { strongly in } C\left(J ; H^{1-r}(\Omega)\right) \\
& \psi_{n} \rightarrow \psi \quad \text { strongly in } C\left(J ; H^{1-r}(\Gamma)\right),
\end{aligned}
$$

for all $r \in(0,1 / 2)$ so that $(u(t), \psi(t)) \in C\left(J ; H^{1-r}(\Omega) \times H^{1-r}(\Gamma)\right)$. These strong convergences entail that, up to subsequences, $u_{n}$ converges to $u$ almost everywhere in $\Omega \times J$ and $\psi_{n}$ converges to $\psi$ almost everywhere in $\Gamma \times J$. Then, using the continuity of $f$ and $g$, (3.39)-(3.40) and (3.33), we easily deduce that, up to subsequences, $f\left(u_{n}\right)$ weakly converges to $f(u)$ in $L^{2}(\Omega \times J)$, while $g\left(\psi_{n}\right)$ weakly converges to $g(\psi)$ in $L^{q+1}(\Gamma \times J)$, and hence in $L^{2}(\Gamma \times J)$, since $q \geq 1$. Thus we can now pass to the limit in equations (3.14) and (3.15) and recover (3.7) and (3.8), respectively.

It remains to pass to the limit in equation (3.16). An integration by parts and (3.37) yield, for any $\zeta \in C_{0}^{\infty}\left(J ; C_{0}^{\infty}\left(\mathbb{R}_{+} ; H^{1}(\Omega)\right)\right)$,

$$
\int_{\tau}^{T}\left(\partial_{t} \eta_{n}^{y}, \zeta\right)_{\mathcal{M}_{-1}} d y=-\int_{\tau}^{T}\left(\eta_{n}^{y}, \partial_{t} \zeta\right)_{\mathcal{M}_{-1}} d y \rightarrow-\int_{\tau}^{T}\left(\eta^{y}, \partial_{t} \zeta\right)_{\mathcal{M}_{-1}} d y
$$

Thus, we have

$$
\partial_{t} \eta_{n}^{t} \rightarrow \partial_{t} \eta^{t} \text { weakly in } L^{2}\left(J ; H_{\nu}^{-1}\left(\mathbb{R}_{+} ; H^{-1}(\Omega)\right)\right),
$$

and that $\eta^{t} \in C\left(J ; H_{\nu}^{-1}\left(\mathbb{R}_{+} ; H^{-1}(\Omega)\right)\right)$. Moreover, on account of (3.38), we have

$$
-\int_{\tau}^{T}\left(\mathbb{T} \eta_{n}^{y}, \zeta\right)_{\mathcal{M}_{-1}} d y=-\int_{\tau}^{T} \nu^{\prime}(s)\left(\eta_{n}^{y}, \zeta\right)_{H^{-1}} d y \rightarrow-\int_{\tau}^{T} \nu^{\prime}(s)\left(\eta^{y}, \zeta\right)_{H^{-1}} d y
$$

Exploiting a density argument and the following distributional equality (see [30])

$$
-\int_{\tau}^{T}\left(\eta^{y}, \partial_{t} \zeta\right)_{\mathcal{M}_{-1}} d y-\int_{\tau}^{T} \nu^{\prime}(s)\left(\eta^{y}, \zeta\right)_{H^{-1}} d y=\int_{\tau}^{t}\left(\partial_{t} \eta^{t}-\mathbb{T} \eta^{y}, \zeta\right)_{\mathcal{M}_{-1}} d y
$$

we also get (3.9) on account of (3.37). The proof is finished.

In the viscous case we can also prove the existence of smoother solutions.

Theorem 3.4 Let $\alpha>0$. Assume (K1)-(K4) and (N0'),(N1), (N2). If $\left(u_{0}, \psi_{0}, \eta_{0}\right) \in \mathcal{H}_{3,2}$ is such that

$$
\eta_{0} \in D(\mathbb{T})
$$


then, for any given initial time $\tau \in \mathbb{R}$, problem $\boldsymbol{P}$ admits at least one solution $(u, \psi, \eta)$ on the time interval J, according to Definition 3.1. In addition, this solution has the following regularity properties

$$
\begin{aligned}
& (u, \psi, \eta) \in L^{\infty}\left(J ; \mathcal{H}_{3,2}\right) \cap W^{1, \infty}\left(J ; \mathcal{H}_{1,0}\right), \\
& \partial_{t t} u \in L^{\infty}\left(J ; H^{-1}(\Omega)\right), \quad \alpha^{1 / 2} \partial_{t t} u \in L^{2}(\Omega \times J), \quad \partial_{t t} \psi \in L^{2}(\Gamma \times J), \\
& \eta \in L^{\infty}(J ; D(\mathbb{T})) .
\end{aligned}
$$

Proof. We use again the approximation scheme developed in the proof of Theorem 3.3. In the sequel of this proof, $c$ will indicate a positive constant which may depend on $\alpha, \beta, c_{f}$, $c_{g}, \Omega, \tau$ and $T$, but is independent of $n$.

More precisely, referring to problem $\mathbf{P}_{n}$, we will consider instead the approximating problem of finding $\left(u_{n}, \psi_{n}, \mu_{n}, \eta_{n}\right)$ of the form (3.12)-(3.13) such that

$$
\begin{aligned}
& \left(\partial_{t t} u_{n}, v\right)+\int_{0}^{\infty} \nu(s)\left(\partial_{t} \eta_{n}(s), v\right) d s=0, \\
& \Upsilon\left(\partial_{t} \mathbf{u}_{n}, \mathbf{v}\right)+\left(f^{\prime}\left(u_{n}\right) \partial_{t} u_{n}, v\right)+\left(g^{\prime}\left(\psi_{n}\right) \partial_{t} \psi_{n}, w\right)_{\Gamma} \\
& +\left(\alpha \partial_{t t} u_{n}, v\right)+\left(\partial_{t t} \psi_{n}, w\right)_{\Gamma}=\left(\partial_{t} \mu_{n}, v\right), \\
& \left(\partial_{t t} \eta_{n}-\mathbb{T} \partial_{t} \eta_{n}, \zeta\right)_{\mathcal{M}_{-1}}=\left(\partial_{t} \mu_{n}, \zeta\right)_{\mathcal{M}_{0}},
\end{aligned}
$$

for every $t \in\left(\tau, t_{n}\right)$ and every $\mathbf{v}=(v, w) \in X_{n}$ and $\zeta \in \mathcal{M}^{n}$, respectively, which satisfies initial conditions (3.17) and

$$
\partial_{t} u_{n}(\tau)=\mathcal{O}_{n} \widetilde{u}_{0}, \quad \partial_{t} \psi_{n}(\tau)=\widetilde{\mathcal{O}}_{n} \widetilde{u}_{0}, \quad \partial_{t} \eta_{n}^{\tau}=\mathcal{Q}_{n} \widetilde{\eta}_{0} .
$$

Here we have set

$$
\widetilde{u}_{0}:=-\int_{0}^{\infty} \nu(s) \eta_{0}(s) d s, \widetilde{\psi}_{0}:=\Delta_{\Gamma} \psi_{0}-\partial_{\mathbf{n}} u_{0}+\beta \psi_{0}+g\left(\psi_{0}\right), \widetilde{\eta}_{0}:=\mathbb{T} \eta_{0}+A_{N} \mu_{0},
$$

where

$$
\mu_{0}=-\alpha \int_{0}^{\infty} \nu(s) \eta_{0}(s) d s-\Delta u_{0}+f\left(u_{0}\right) .
$$

Note that if $\left(u_{0}, \psi_{0}, \eta_{0}\right)$ satisfies the assumptions of Theorem 3.4 , then $\left(\widetilde{u}_{0}, \widetilde{\psi}_{0}, \widetilde{\eta}_{0}\right) \in \mathcal{H}_{1,0}$ (in fact, owing to the boundedness of the projectors $\mathcal{O}_{n}, \widetilde{\mathcal{O}}_{n}$ and $\mathcal{Q}_{n}$ on the corresponding subspaces, $\left\|\left(\partial_{t} u_{n}(\tau), \partial_{t} \psi_{n}(\tau), \partial_{t} \eta_{n}^{\tau}\right)\right\|_{\mathcal{H}_{1,0}} \leq Q\left(\left\|\left(u_{0}, \psi_{0}, \eta_{0}\right)\right\|_{\mathcal{H}_{3,2}}\right)$, for some monotone increasing function $Q)$.

Thus the previous Galerkin scheme can be used. Arguing as for $\mathbf{P}_{n}$ and recalling (N0'), for any fixed $n \in \mathbb{N}$, we have a local unique (maximal) solution $\left(u_{n}, \psi_{n}, \eta_{n}\right) \in C^{3}\left(\left[\tau, t_{n}\right] ; \mathcal{H}_{2,2}\right)$. Also, if we integrate equations (3.45)-(3.46) with respect to time from $\tau$ to $t \in\left(\tau, t_{n}\right)$, reasoning as in the proof of Theorem 3.3, we can find uniform bounds such as the ones in (3.29)-(3.30). To obtain higher-order estimates, we proceed for the sake of simplicity in a formal way by removing the index $n$ and setting

$$
\widetilde{u}=\partial_{t} u, \quad \widetilde{\psi}=\partial_{t} \psi, \quad \widetilde{\mu}=\partial_{t} \mu, \quad \widetilde{\eta}^{t}=\partial_{t} \eta^{t},
$$


so that $(\widetilde{u}, \widetilde{\psi}, \widetilde{\eta})$ solves

$$
\begin{aligned}
& \left(\partial_{t} \widetilde{u}, v\right)_{H^{-1}, H^{1}}+\int_{0}^{\infty} \nu(s)\left(\widetilde{\eta}^{t}(s), v\right)_{H^{-1}, H^{1}} d s=0, \quad \forall v \in H^{1}(\Omega), \\
& \Upsilon(\widetilde{\mathbf{u}}, \mathbf{v})+\left(f^{\prime}(u) \widetilde{u}, v\right)+\left(g^{\prime}(\psi) \widetilde{\psi}, w\right)_{\Gamma}+\left(\alpha \partial_{t} \widetilde{u}, v\right)+\left(\partial_{t} \widetilde{\psi}, w\right)_{\Gamma} \\
& =(\widetilde{\mu}, v)_{H^{-1}, H^{1}}, \quad \forall \mathbf{v} \in \mathbb{V}_{1}, \\
& \left(\partial_{t} \widetilde{\eta}-\mathbb{T} \widetilde{\eta}, \zeta\right)_{\mathcal{M}_{-1}}=(\widetilde{\mu}, \zeta)_{\mathcal{M}_{0}}, \quad \forall \zeta \in \mathcal{M}_{1},
\end{aligned}
$$

with initial conditions

$$
\widetilde{u}(\tau)=\widetilde{u}_{0}, \quad \widetilde{\psi}(\tau)=\widetilde{\psi}_{0}, \quad \widetilde{\eta}^{\tau}=\widetilde{\eta}_{0} .
$$

We now take $v=\widetilde{\mu}(t)$ in equation (3.51), $\bar{v}=\partial_{t} \widetilde{u}(t)$ and $w=\partial_{t} \widetilde{\psi}(t)$ in (3.52), and $\zeta=\widetilde{\eta}^{t}$ in equation (3.53). Adding together the resulting identities, we obtain

$$
\begin{aligned}
& \frac{1}{2} \frac{d}{d t} \widetilde{E}(t)-\int_{0}^{\infty} \nu^{\prime}(s)\left\|\widetilde{\eta}^{t}(s)\right\|_{H^{-1}}^{2} d s+\alpha\left\|\partial_{t} \widetilde{u}(t)\right\|^{2}+\left\|\partial_{t} \widetilde{\psi}(t)\right\|_{\Gamma}^{2} \\
& =\left(f^{\prime}(u(t)) \widetilde{u}(t), \partial_{t} \widetilde{u}(t)\right)+\left(g^{\prime}(\psi(t)) \widetilde{\psi}(t), \partial_{t} \widetilde{\psi}(t)\right)_{\Gamma}, \quad \forall t \in J
\end{aligned}
$$

where we have set

$$
\widetilde{E}(t)=\|\nabla \widetilde{u}(t)\|^{2}+\left\|\nabla_{\Gamma} \widetilde{\psi}(t)\right\|_{\Gamma}^{2}+\beta\|\widetilde{\psi}(t)\|_{\Gamma}^{2}+\left\|\widetilde{\eta}^{t}\right\|_{\mathcal{M}_{-1}}^{2} .
$$

By standard Hölder, Young and Sobolev inequalities, recalling (N2), we have

$$
\begin{aligned}
& \left(f^{\prime}(u(t)) \widetilde{u}(t), \partial_{t} \widetilde{u}(t)\right)+\left(g^{\prime}(\psi(t)) \widetilde{\psi}(t), \partial_{t} \widetilde{\psi}(t)\right)_{\Gamma} \\
& \leq \frac{\alpha}{2}\left\|\partial_{t} \widetilde{u}(t)\right\|^{2}+\frac{1}{2}\left\|\partial_{t} \widetilde{\psi}(t)\right\|_{\Gamma} \\
& +c\left(\left(1+\|u(t)\|_{H^{1}}^{2}\right)\|\widetilde{u}(t)\|_{H^{1}}^{2}+\left(1+\|\psi(t)\|_{H^{1}(\Gamma)}^{2}\right)\|\widetilde{\psi}(t)\|_{H^{1}(\Gamma)}^{2}\right),
\end{aligned}
$$

for all $t \in J$. Then, using the known bound on $u$ and $\psi$ (see (3.29)), on account of (3.56), integrating the inequality (3.55) with respect to time, we obtain, for all $t \in J$,

$$
\begin{aligned}
& \widetilde{E}(t)-2 \int_{\tau}^{t} \int_{0}^{\infty} \nu^{\prime}(s)\left\|\widetilde{\eta}^{y}(y)\right\|_{H^{-1}}^{2} d s d y+2 \alpha \int_{\tau}^{t}\left\|\partial_{t} \widetilde{u}(y)\right\|^{2} d y+2 \int_{\tau}^{t}\left\|\partial_{t} \widetilde{\psi}(y)\right\|_{\Gamma}^{2} d y \\
& \leq \widetilde{E}(\tau)+c \int_{\tau}^{t}\left(\|\widetilde{u}(y)\|_{H^{1}}^{2}+\|\widetilde{\psi}(y)\|_{H^{1}(\Gamma)}^{2}\right) d y .
\end{aligned}
$$

Then the standard Gronwall lemma gives

$$
\left\|\left(\widetilde{u}(t), \widetilde{\psi}(t), \widetilde{\eta}^{t}\right)\right\|_{\mathcal{H}_{1,0}} \leq c, \quad \forall t \in J
$$


In addition, we have (cf. also (3.31))

$$
\sqrt{\alpha}\left\|\partial_{t} \widetilde{u}\right\|_{L^{2}(\Omega \times J)}+\left\|\partial_{t} \widetilde{\psi}\right\|_{L^{2}(\Gamma \times J)}+\|\widetilde{\eta}\|_{L^{2}\left(J ; L_{\nu^{\prime}}^{2}\left(\mathbb{R}_{+} ; H^{-1}(\Omega)\right)\right)} \leq c .
$$

Here $c$ also depends on $\left\|\left(u_{0}, \psi_{0}, \eta_{0}\right)\right\|_{\mathcal{H}_{3,2}}$ and on $\left\|\mathbb{T} \eta_{0}\right\|_{\mathcal{M}_{-1}}$.

Let us now consider equation (3.9) (or, more precisely, (3.16)), then set $\bar{\mu}(t)=\mu(t)-$ $\langle\mu(t)\rangle$ and take $\zeta=A_{N} \bar{\mu}(t)$. On account of (3.30) and (3.58), we obtain

$$
\begin{aligned}
\|\nabla \mu(t)\|^{2} & =\int_{0}^{\infty} \nu(s)\left(\bar{\mu}(t), \partial_{t} \eta^{t}(s)\right) d s-\int_{0}^{\infty} \nu^{\prime}(s)\left(\bar{\mu}(t), \eta^{t}(s)\right) d s \\
& \leq c_{\varepsilon}\left(\left\|\partial_{t} \eta^{t}\right\|_{\mathcal{M}_{-1}}^{2}-\int_{0}^{\infty} \nu^{\prime}(s)\left\|\eta^{t}(s)\right\|_{H^{-1}}^{2} d s\right)+\varepsilon\|\nabla \mu(t)\|^{2} \\
& \leq c_{\varepsilon}\left(1-\int_{0}^{\infty} \nu^{\prime}(s)\left\|\eta^{t}(s)\right\|_{H^{-1}}^{2} d s\right)+\varepsilon\|\nabla \mu(t)\|^{2}, \\
& \leq c_{\varepsilon}+\varepsilon\|\nabla \mu(t)\|^{2},
\end{aligned}
$$

for all $t \in J$ and every $\varepsilon>0$, where $c_{\varepsilon}$ is a positive constant that depends also on $\varepsilon$. Then, recalling (3.34), we deduce the bound

$$
\|\mu(t)\|_{H^{1}(\Omega)} \leq c, \quad t \in J .
$$

Thus, reasoning as in the proof of Theorem 3.3, it is not difficult to realize that we can find a solution $(u, \psi, \eta) \in W^{1, \infty}\left(J ; \mathcal{H}_{1,0}\right)$ to problem $\mathbf{P}$ which satisfies (3.43). Moreover, due to (3.60), we also have $\mu \in L^{\infty}\left(J ; H^{1}(\Omega)\right)$. On the other hand, recalling (3.34) and (3.41), we have that $\psi$ fulfills (3.44) thanks to the representation formula (see [30]). Therefore, we are left to prove that $(u, \psi, \eta) \in L^{\infty}\left(J ; \mathbb{V}_{3} \times \mathcal{M}_{1}\right)$. First of all observe that from equation (3.7) we deduce

$$
\left\|\eta^{t}\right\|_{\mathcal{M}_{1}}^{2}=-\int_{0}^{\infty} \nu(s)\left[\left(\nabla \partial_{t} u(t), \nabla \eta^{t}(s)\right)+\left(\partial_{t} u(t), \eta^{t}(s)\right)\right] d s,
$$

that is, $\eta \in L^{\infty}\left(J ; \mathcal{M}_{1}\right)$. Finally, we consider the variational identity (3.8). On account of the regularity properties of $\partial_{t} u, \partial_{t} \psi$ and $\mu$, we can use the results in [39, Appendix A] (see also $\left[2\right.$, Lemma 2.2]) to infer that $(u, \psi) \in L^{\infty}\left(J ; \mathbb{V}_{3}\right)$. This concludes the proof.

The uniqueness of solutions to problem $\mathbf{P}$ in the viscous case follows from the following continuous dependence estimate.

Lemma 3.5 Let the assumptions of Theorem 3.3 hold and assume that $\alpha>0$. Let $\left(u_{i}, \psi_{i}, \eta_{i}\right)$ be two solutions to $\boldsymbol{P}$ corresponding to the initial data $\left(u_{0 i}, \psi_{0 i}, \eta_{0 i}\right) \in \mathcal{H}_{1,0}, i=1,2$. Then, for any $t \geq \tau$, the following estimate holds:

$$
\begin{aligned}
& \left\|\left(u_{1}-u_{2}\right)(t)\right\|_{H^{1}}^{2}+\left\|\left(\psi_{1}-\psi_{2}\right)(t)\right\|_{H^{1}(\Gamma)}^{2}+\left\|\eta_{1}^{t}-\eta_{2}^{t}\right\|_{\mathcal{M}_{-1}}^{2} \\
& +\int_{\tau}^{t}\left(\alpha\left\|\partial_{t}\left(u_{1}-u_{2}\right)(y)\right\|^{2}+\left\|\partial_{t}\left(\psi_{1}-\psi_{2}\right)(y)\right\|_{\Gamma}^{2}\right) d y \\
& \leq C e^{C(t-\tau)}\left(\left\|u_{01}-u_{02}\right\|_{H^{1}}^{2}+\left\|\psi_{01}-\psi_{02}\right\|_{H^{1}(\Gamma)}^{2}+\left\|\eta_{01}-\eta_{02}\right\|_{\mathcal{M}_{-1}}^{2}\right),
\end{aligned}
$$


where $C$ is a positive constant depending on the norms of the initial data in $\mathcal{H}_{1,0}$, on $\Omega, \Gamma$, $\beta \alpha$, but is independent of time.

Proof. We first prove estimate (3.62) for the smoother solutions constructed in Theorem 3.4. Thus, we assume that $\left(u_{0 i}, \psi_{0 i}, \eta_{0 i}\right), i=1,2$, satisfy the assumptions of Theorem 3.4. Then set

$$
u=u_{1}-u_{2}, \quad \psi=\psi_{1}-\psi_{2}, \quad \eta=\eta_{1}-\eta_{2}
$$

where $\left(u_{i}, \psi_{i}, \eta_{i}\right)$ is a solution corresponding to $\left(u_{0 i}, \psi_{0 i}, \eta_{0 i}\right)$. Then, $(u, \psi, \eta)$ solves

$$
\begin{aligned}
& \quad\left(\partial_{t} u, v\right)_{H^{-1}, H^{1}}+\int_{0}^{\infty} \nu(s)\left(\eta^{t}(s), v\right)_{H^{-1}, H^{1}} d s=0, \quad \forall v \in H^{1}(\Omega), \\
& \Upsilon(\mathbf{u}, \mathbf{v})+\left(f\left(u_{1}\right)-f\left(u_{2}\right), v\right)+\left(g\left(\psi_{1}\right)-g\left(\psi_{1}\right), w\right)_{\Gamma}+\left(\alpha \partial_{t} u, v\right)+\left(\partial_{t} \psi, w\right)_{\Gamma} \\
& \quad=(\mu, v)_{H^{-1}, H^{1}}, \quad \forall \mathbf{v}=(v, w) \in \mathbb{V}_{1}, \\
& \quad\left(\partial_{t} \eta-\mathbb{T} \eta, \zeta\right)_{\mathcal{M}_{-1}}=(\mu, \zeta)_{\mathcal{M}_{0}}, \quad \forall \zeta \in \mathcal{M}_{0},
\end{aligned}
$$

with initial condition

$$
u(\tau)=u_{0}:=u_{01}-u_{02}, \quad \psi(\tau)=\psi_{0}:=\psi_{01}-\psi_{02}, \quad \eta^{\tau}=\eta_{0}:=\eta_{01}-\eta_{02} .
$$

Taking $v=\mu(t)$ and $\mathbf{v}=\left(\partial_{t} u(t), \partial_{t} \psi(t)\right)$ in (3.63) and (3.64), respectively, then adding the obtained relations together, we deduce, for all $t \in J$,

$$
\begin{aligned}
& \frac{1}{2} \frac{d}{d t}\left(\|\nabla u(t)\|^{2}+\left\|\nabla_{\Gamma} \psi(t)\right\|_{\Gamma}^{2}+\beta\|\psi(t)\|_{\Gamma}^{2}\right) \\
& +\alpha\left\|\partial_{t} u(t)\right\|^{2}+\left\|\partial_{t} \psi(t)\right\|_{\Gamma}^{2}+\int_{0}^{\infty} \nu(s)\left(\eta_{m n}^{t}(s), \mu\right) d s \\
& =-\left(f\left(u_{1}(t)\right)-f\left(u_{2}(t)\right), \partial_{t} u(t)\right)+\left(g\left(\psi_{1}(t)\right)-g\left(\psi_{2}(t)\right), \partial_{t} \psi(t)\right)_{\Gamma} .
\end{aligned}
$$

Moreover, take $\zeta=\eta^{t}$ in (3.65). This gives, for all $t \in J$,

$$
\frac{1}{2} \frac{d}{d t}\left\|\eta^{t}\right\|_{\mathcal{M}_{-1}}^{2}-\int_{0}^{\infty} \nu^{\prime}(s)\left\|\eta^{t}(s)\right\|_{H^{-1}}^{2} d s=\int_{0}^{\infty} \nu(s)\left(\mu(t), \eta^{t}(s)\right) d s .
$$

Adding (3.66) to (3.67), we thus obtain the differential inequality

$$
\begin{aligned}
& \frac{d}{d t} E(t)+2 \alpha\left\|\partial_{t} u(t)\right\|^{2}+2\left\|\partial_{t} \psi(t)\right\|_{\Gamma}^{2} \\
& \leq R(t):=-2\left(f\left(u_{1}(t)\right)-f\left(u_{2}(t)\right), \partial_{t} u(t)\right)+2\left(g\left(\psi_{1}(t)\right)-g\left(\psi_{2}(t)\right), \partial_{t} \psi(t)\right)_{\Gamma},
\end{aligned}
$$

for all $t \in J$, where

$$
E(t):=\|\nabla u(t)\|^{2}+\left\|\nabla_{\Gamma} \psi(t)\right\|_{\Gamma}^{2}+\beta\|\psi(t)\|_{\Gamma}^{2}+\left\|\eta^{t}\right\|_{\mathcal{M}_{-1}}^{2} .
$$

Observe that, thanks to (N2), there holds

$$
\begin{aligned}
& \left|f\left(y_{1}\right)-f\left(y_{2}\right)\right| \leq c_{f}\left(1+\left|y_{1}\right|^{2}+\left|y_{2}\right|^{2}\right)\left|y_{1}-y_{2}\right| \\
& \left|g\left(y_{1}\right)-g\left(y_{2}\right)\right| \leq c_{g}\left(1+\left|y_{1}\right|^{q}+\left|y_{2}\right|^{q}\right)\left|y_{1}-y_{2}\right|
\end{aligned}
$$


Thus, using standard Young, Hölder and Sobolev inequalities, we can estimate the terms on the right-hand side of (3.68) as follows:

$$
\begin{aligned}
R(t) & \leq \alpha\left\|\partial_{t} u(t)\right\|^{2}+\left\|\partial_{t} \psi(t)\right\|_{\Gamma}^{2}+c_{\alpha}\left(1+\left\|u_{1}(t)\right\|_{L^{6}}^{4}+\left\|u_{2}(t)\right\|_{L^{6}}^{4}\right)\|u(t)\|_{H^{1}}^{2} \\
& +c\left(1+\left\|\psi_{1}(t)\right\|_{L^{3 q}(\Gamma)}^{2 q}+\left\|\psi_{2}(t)\right\|_{L^{3 q}(\Gamma)}^{2 q}\right)\|\psi(t)\|_{H^{1}(\Gamma)}^{2}, \quad \forall t \in J,
\end{aligned}
$$

for some positive constants $c$ and $c_{\alpha}$.

Thus, combining (3.68) with (3.71) and recalling that $\left(u_{i}, \psi_{i}\right)$ satisfy $(3.11)$ for $i=1,2$, we deduce, for all $t \in J$,

$$
\frac{d}{d t} E(t)+\alpha\left\|\partial_{t} u(t)\right\|^{2}+\left\|\partial_{t} \psi(t)\right\|_{\Gamma}^{2} \leq C\left(\|u(t)\|_{H^{1}}^{2}+\|\psi(t)\|_{H^{1}(\Gamma)}^{2}\right)
$$

for some positive constant $C$ which depends only on the norms of the initial data in $\mathcal{H}_{1,0}$. Therefore, an application of standard Gronwall's inequality to (3.72) yields

$$
E(t) \leq C e^{C(t-\tau)} E(\tau)
$$

for all $t \in J$. Then, an integration with respect to time from $\tau$ to $t$ of (3.72) combined with (3.73) entails the desired estimate (3.62). To conclude, it is not difficult to realize that such an estimate still holds for the solutions given by Theorem 3.3 by employing standard approximation arguments (note that the constants above do not depend on the extra-assumptions of Theorem 3.4). The proof of lemma is finished.

The following is a straightforward consequence of the above results.

Corollary 3.6 Let the assumptions of Lemma 3.5 hold. Then, problem $\boldsymbol{P}$ admits a unique solution $(u, \psi, \eta) \in C\left([\tau, T] ; \mathcal{H}_{1,0}\right)$, for all $T>\tau$, and the nonlinear operator $S(t): \mathcal{H}_{1,0} \rightarrow$ $\mathcal{H}_{1,0}$ defined by

$$
S(t)\left(u_{0}, \psi_{0}, \eta_{0}\right)=\left(u(t), \psi(t), \eta^{t}\right), \quad \forall t \geq \tau,
$$

is a strongly continuous semigroup.

Remark 3.7 We recall that the issue of uniqueness in the non-viscous case $(\alpha=0)$ seems particularly hard (see [5, 27, 28]).

\section{Global attractors}

Our aim in this section is to prove that $S(t)$ possesses the global attractor in the phase space $\mathcal{H}_{1,0}^{M}$, for some given $M \geq 0$. This choice is due to the constraints (2.9). Thus the main result of this section is

Theorem 4.1 Let $\alpha>0$. Assume (K1),(K3)-(K5) and (N0'),(N1),(N2'). Then, $\left(\mathcal{H}_{1,0}^{M}, S(t)\right)$ possesses the connected global attractor $\mathcal{A}_{M}$ which is bounded in $\mathcal{H}_{3,2}$. 
We begin to prove the existence of a bounded absorbing set in $\mathcal{H}_{1,0}^{M}$ for $S(t)$.

Lemma 4.2 Let the assumptions of Theorem 3.3 be satisfied and, in addition, replace (K2) with (K5). Then, for any given initial data $\left(u_{0}, \psi_{0}, \eta_{0}\right) \in \mathcal{H}_{1,0}^{M}$, there exists a solution $(u, \psi, \eta)$ to $\boldsymbol{P}$ which satisfies the uniform estimate

$$
\begin{aligned}
& \left\|\left(u(t), \psi(t), \eta^{t}\right)\right\|_{\mathcal{H}_{1,0}}^{2}+\int_{t}^{t+1}\left(\alpha\left\|\partial_{t} u(y)\right\|^{2}+\left\|\partial_{t} \psi(y)\right\|_{\Gamma}^{2}\right) d y \\
& \leq Q\left(\left\|\left(u_{0}, \psi_{0}, \eta_{0}\right)\right\|_{\mathcal{H}_{1,0}}\right) e^{-\rho(t-\tau)}+C_{M},
\end{aligned}
$$

for each $t \geq \tau$. Here $\rho C_{M}$ are positive constants which are independent of $t, \alpha$ and of the initial data. $Q$ is a monotone non-negative non-decreasing function which is independent of $t$ and $\alpha$.

Proof. We will work again within the approximation scheme used in the proof of Theorem 3.3. Consider the functional

$$
\mathcal{Y}_{n}(t)=E_{n}(t)+\varepsilon\left(\alpha\left\|\bar{u}_{n}(t)\right\|^{2}+\left\|\bar{\psi}_{n}(t)\right\|_{\Gamma}^{2}\right)-2 \varepsilon \int_{0}^{\infty} \nu(s)\left(\bar{u}_{n}(t), A_{N}^{-1} \eta_{n}^{t}(s)\right) d s,
$$

where $\varepsilon \in(0, \lambda)$ is sufficiently small. Here we have set

$$
\bar{u}_{n}(t):=u_{n}(t)-\left\langle u_{n}(\tau)\right\rangle, \quad \bar{\psi}_{n}(t):=\psi_{n}(t)-\left\langle u_{n}(\tau)\right\rangle .
$$

Note that $\left\langle\bar{u}_{n}(t)\right\rangle=\left\langle\partial_{t} \bar{u}_{n}(t)\right\rangle=0$, for all $t>\tau$. We observe preliminarily, on account of equations (3.14)-(3.16), that

$$
\begin{aligned}
& -\frac{d}{d t} \int_{0}^{\infty} \nu(s)\left(\bar{u}_{n}(t), A_{N}^{-1} \eta_{n}^{t}(s)\right) d s \\
& =\left\|\partial_{t} u_{n}(t)\right\|_{H^{-1}}^{2}-\int_{0}^{\infty} \nu(s)\left(\bar{u}_{n}(t), A_{N}^{-1} \partial_{t} \eta_{n}^{t}(s)\right) d s \\
& =\left\|\partial_{t} u_{n}(t)\right\|_{H^{-1}}^{2}-\int_{0}^{\infty} \nu^{\prime}(s)\left(\bar{u}_{n}(t), A_{N}^{-1} \eta_{n}^{t}(s)\right) d s-\int_{0}^{\infty} \nu(s)\left(\bar{u}_{n}(t), \mu_{n}(t)\right) d s \\
& =\left\|\partial_{t} u_{n}(t)\right\|_{H^{-1}}^{2}-\int_{0}^{\infty} \nu^{\prime}(s)\left(\bar{u}_{n}(t), A_{N}^{-1} \eta_{n}^{t}(s)\right) d s-\frac{1}{2} \frac{d}{d t}\left[\alpha\left\|_{n}\right\|^{2}+\left\|\bar{\psi}_{n}(t)\right\|_{\Gamma}^{2}\right] \\
& -\left\|\nabla \bar{u}_{n}(t)\right\|^{2}-\left\|\nabla_{\Gamma} \bar{\psi}_{n}(t)\right\|_{\Gamma}^{2}-\beta\left(\bar{\psi}_{n}(t), \psi_{n}(t)\right)_{\Gamma} \\
& -\left(f\left(u_{n}(t)\right), \bar{u}_{n}(t)\right)-\left(g\left(\psi_{n}(t)\right), \bar{\psi}_{n}(t)\right)_{\Gamma} .
\end{aligned}
$$

Differentiating the expression for $\mathcal{Y}_{n}(t)$ with respect to time and recalling (3.26), we deduce

$$
\frac{d}{d t} \mathcal{Y}_{n}(t)+\xi \mathcal{Y}_{n}(t)-2 \int_{0}^{\infty} \nu^{\prime}(s)\left\|\eta_{n}^{t}(s)\right\|_{H^{-1}}^{2} d s=h_{n}(t)
$$


Here $\xi \in(0, \varepsilon)$ and

$$
\begin{aligned}
h_{n}(t) & =-2 \alpha\left\|\partial_{t} u_{n}(t)\right\|^{2}+2 \varepsilon\left\|\partial_{t} u_{n}(t)\right\|_{H^{-1}}^{2}-2\left\|\partial_{t} \psi_{n}(t)\right\|_{\Gamma}^{2} \\
& -2 \varepsilon \int_{0}^{\infty} \nu^{\prime}(s)\left(\bar{u}_{n}(t), A_{N}^{-1} \eta_{n}^{t}(s)\right) d s \\
& -2 \xi\left[\left(f\left(u_{n}(t)\right) \bar{u}_{n}(t)-F\left(u_{n}(t)\right), 1\right)+\left(g\left(\psi_{n}(t)\right) \bar{\psi}_{n}(t)-G\left(\psi_{n}(t)\right), 1\right)_{\Gamma}\right] \\
& -2(\varepsilon-\xi)\left[\left(f\left(u_{n}(t)\right), \bar{u}_{n}(t)\right)+\left(g\left(\psi_{n}(t)\right), \bar{\psi}_{n}(t)\right)_{\Gamma}\right]+\xi\left\|\eta_{n}^{t}\right\|_{\mathcal{M}_{-1}}^{2} \\
& -(2 \varepsilon-\xi)\left(\left\|\nabla \bar{u}_{n}(t)\right\|^{2}+\left\|\nabla_{\Gamma} \bar{\psi}_{n}(t)\right\|_{\Gamma}^{2}\right)+\left(\xi\left\|\psi_{n}(t)\right\|_{\Gamma}^{2}-2 \varepsilon \beta\left(\bar{\psi}_{n}(t), \psi_{n}(t)\right)_{\Gamma}\right) \\
& +\xi \varepsilon\left(\alpha\left\|\bar{u}_{n}(t)\right\|^{2}+\left\|\bar{\psi}_{n}(t)\right\|_{\Gamma}^{2}\right)-2 \varepsilon \xi \int_{0}^{\infty} \nu(s)\left(\bar{u}_{n}(t), A_{N}^{-1} \eta_{n}^{t}(s)\right) d s+\xi \widetilde{C} .
\end{aligned}
$$

Let us first notice that Hölder and Young inequalities yield, on account of the fact $\left|\left\langle u_{n}(\tau)\right\rangle\right| \leq$ $|\langle u(\tau)\rangle| \leq M$,

$$
\xi\left\|\psi_{n}\right\|_{\Gamma}^{2}-2 \varepsilon \beta\left(\bar{\psi}_{n}, \psi_{n}\right)_{\Gamma} \leq-(\varepsilon \beta-\xi)\left\|\bar{\psi}_{n}\right\|_{\Gamma}^{2}+C_{M},
$$

where $C_{M}>0$ depends obviously on $M, \xi, \varepsilon,|\Omega|$ and $|\Gamma|$, but is independent of $n, t$ and $\alpha$. Here $|\mathcal{S}|$ denotes the $N$-dimensional Lebesgue measure of a set $\mathcal{S} \subset \mathbb{R}^{N}$. On the other hand, due to assumption (N1), we have, for all $y \in \mathbb{R}$,

$$
\begin{aligned}
& F(y)-C_{F, M_{\tau}} \leq c_{f}\left(y-M_{\tau}\right)^{2}+f(y)\left(y-M_{\tau}\right), \\
& \frac{1}{2}|F(s)| \leq f(y)\left(y-M_{\tau}\right)+C_{F, M_{\tau}}^{\prime}, \\
& G(y)-C_{G, M_{\tau}} \leq c_{g}\left(y-M_{\tau}\right)^{2}+g(y)\left(y-M_{\tau}\right), \\
& \frac{1}{2}|G(y)| \leq g(y)\left(y-M_{\tau}\right)+C_{G, M_{\tau}}^{\prime},
\end{aligned}
$$

where all the positive constants in (4.7)-(4.10) are sufficiently large and we have set $M_{\tau}:=$ $\langle u(\tau)\rangle$. Consequently, using (4.6)-(4.9) and the Poincaré-Wirtinger inequality (i.e., $\left\|\bar{u}_{n}\right\| \leq$ $\left.\sqrt{C_{\Omega}}\left\|\nabla \bar{u}_{n}\right\|\right)$, it is possible to estimate $h_{n}$ as follows:

$$
\begin{aligned}
h_{n} & \leq-2 \alpha\left\|\partial_{t} u_{n}\right\|^{2}+2 \varepsilon\left\|\partial_{t} u_{n}\right\|_{H^{-1}}^{2}-2\left\|\partial_{t} \psi_{n}\right\|_{\Gamma}^{2} \\
& -(\varepsilon-\xi)\left[\left(\left|F\left(u_{n}\right)\right|, 1\right)+\left(\left|G\left(\psi_{n}\right)\right|, 1\right)_{\Gamma}\right] \\
& -\left[\varepsilon \beta-\xi\left(1+2 c_{g}+\varepsilon\right)\right]\left\|\bar{\psi}_{n}\right\|_{\Gamma}^{2}-\left[2 \varepsilon-\xi\left(1+2 c_{f} C_{\Omega}+\varepsilon \alpha C_{\Omega}\right)\right]\left\|\nabla \bar{u}_{n}\right\|^{2} \\
& -(2 \varepsilon-\xi)\left\|\nabla_{\Gamma} \bar{\psi}_{n}\right\|_{\Gamma}^{2}-2 \varepsilon \int_{0}^{\infty} \nu^{\prime}(s)\left(\bar{u}_{n}, A_{N}^{-1} \eta_{n}(s)\right) d s \\
& -2 \varepsilon \xi \int_{0}^{\infty} \nu(s)\left(\bar{u}_{n}, A_{N}^{-1} \eta_{n}(s)\right) d s+\xi\left\|\eta_{n}\right\|_{\mathcal{M}_{-1}}^{2}+C_{M} .
\end{aligned}
$$


It remains to estimate the integral terms on the right-hand side of (4.11). Employing the Poincaré-Wirtinger inequality repeatedly, in light of (K4), we have

$$
\begin{aligned}
-2 \varepsilon \int_{0}^{\infty} \nu^{\prime}(s)\left(\bar{u}_{n}, A_{N}^{-1} \eta_{n}(s)\right) d s & =-2 \varepsilon \int_{0}^{\infty} \nu^{\prime}(s)\left(A_{N}^{-1 / 2} \bar{u}_{n}, A_{N}^{-1 / 2} \eta_{n}(s)\right) d s \\
& \leq-\varepsilon \int_{0}^{\infty} \nu^{\prime}(s)\left(\frac{\left\|\nabla \bar{u}_{n}\right\|^{2}}{\nu_{0}}+C_{\Omega}^{4} \nu_{0}\left\|\eta_{n}(s)\right\|_{H^{-1}}^{2}\right) d s \\
& \leq \varepsilon\left\|\nabla \bar{u}_{n}\right\|^{2}-\varepsilon C_{\Omega}^{4} \nu_{0} \int_{0}^{\infty} \nu^{\prime}(s)\left\|\eta_{n}(s)\right\|_{H^{-1}}^{2} d s .
\end{aligned}
$$

Moreover, on account of (K3) and (3.31), it is straightforward to check that

$$
\begin{aligned}
& -2 \varepsilon \xi \int_{0}^{\infty} \nu(s)\left(\bar{u}_{n}, A_{N}^{-1} \eta_{n}(s)\right) d s+\xi\left\|\eta_{n}\right\|_{\mathcal{M}_{-1}}^{2}+2 \varepsilon\left\|\partial_{t} u_{n}\right\|_{H^{-1}}^{2} \\
& \leq \varepsilon \xi C_{\Omega}^{4}\left\|\nabla \bar{u}_{n}\right\|^{2}+[\xi(\varepsilon+1)+2 \varepsilon]\left\|\eta_{n}\right\|_{\mathcal{M}_{-1}}^{2} .
\end{aligned}
$$

Thus, exploiting now inequality (K5), it follows from (4.4), (4.11)-(4.13) that we can fix the parameters $\xi, \varepsilon$ small enough in order to find three positive constants $\xi_{1}, \xi_{2}, \xi_{3}$ such that

$$
\begin{aligned}
& \frac{d}{d t} \mathcal{Y}_{n}(t)+\xi_{1} \mathcal{Y}_{n}(t)+\xi_{2}\left\|\eta_{n}^{t}\right\|_{\mathcal{M}_{-1}}^{2}+2 \alpha\left\|\partial_{t} u_{n}(t)\right\|^{2}+2\left\|\partial_{t} \psi_{n}(t)\right\|_{\Gamma}^{2} \\
& +\xi_{3}\left(\left\|\nabla \bar{u}_{n}(t)\right\|^{2}+\left\|\nabla_{\Gamma} \bar{\psi}_{n}(t)\right\|_{\Gamma}^{2}+\left\|\bar{\psi}_{n}(t)\right\|_{\Gamma}^{2}\right) \\
& \leq C_{M} .
\end{aligned}
$$

Note that $C_{M}$ depends on $M, \xi, \varepsilon$ as well as on the other physical parameters of the problem, but is independent of time, $n, \alpha$ and initial data. Besides, one can easily check that, for all $t \geq \tau$,

$$
C_{1}\left\|\left(u_{n}(t), \psi_{n}(t), \eta_{n}^{t}\right)\right\|_{\mathcal{H}_{1,0}}^{2}-C_{2} \leq \mathcal{Y}_{n}(t) \leq Q\left(\left\|\left(u_{n}(t), \psi_{n}(t), \eta_{n}^{t}\right)\right\|_{\mathcal{H}_{1,0}}\right),
$$

for some positive constants $C_{1}, C_{2}$ and monotone non-negative non-decreasing function $Q$ independent of $n$ and $t$. Applying Gronwall's inequality (see, e.g., [26, Lemma 2.5]) to (4.14), then integrating (4.14) and using (4.15), we infer the uniform estimate

$$
\begin{aligned}
& \left\|\left(u_{n}(t), \psi_{n}(t), \eta_{n}^{t}\right)\right\|_{\mathcal{H}_{1,0}}^{2}+\int_{t}^{t+1}\left(\alpha\left\|\partial_{t} u_{n}(y)\right\|^{2}+\left\|\partial_{t} \psi_{n}(y)\right\|_{\Gamma}^{2}\right) d y \\
& \leq Q\left(\left\|\left(u_{0}, \psi_{0}, \eta_{0}\right)\right\|_{\mathcal{H}_{1,0}}\right) e^{-\rho(t-\tau)}+C_{M}, \quad \forall t \geq \tau .
\end{aligned}
$$

Thus, by the lower semicontinuity of the norms, we easily obtain estimate (4.1).

As a consequence we have the following

Proposition 4.3 Let the assumptions of Lemma 3.5 hold. There exists $R_{0}>0$ (independent of time, $\alpha$ and initial data) such $S(t)$ possesses an absorbing ball $\mathcal{B}_{1,0}^{M}\left(R_{0}\right) \subset \mathcal{H}_{1,0}^{M}$, which 
is bounded in $\mathcal{H}_{1,0}^{M}$ independently of $\alpha$. More precisely, for any bounded set $\mathcal{B} \subset \mathcal{H}_{1,0}^{M}$, there exists a time $t_{0}=t_{0}(\mathcal{B})>0$ such that $S(t) \mathcal{B} \subset \mathcal{B}_{1,0}^{M}$, for all $t \geq t_{0}$. Moreover, for every $R>0$, there exists $C_{0}=C_{0}(R) \geq 0$, independent of $\alpha$, such that, for any $\Theta_{0}=\left(u_{0}, \psi_{0}, \eta_{0}\right) \in$ $\mathcal{B}_{\mathcal{H}_{1,0}}(R)$,

$$
\sup _{t \geq \tau}\left\|S(t) \Theta_{0}\right\|_{\mathcal{H}_{1,0}}+\int_{\tau}^{\infty}\left(\alpha\left\|\partial_{t} u(y)\right\|^{2}+\left\|\partial_{t} \psi(y)\right\|_{\Gamma}^{2}\right) d y \leq C_{0},
$$

where $\mathcal{B}_{\mathcal{H}_{1,0}}(R)$ denotes the ball in $\mathcal{H}_{1,0}$ of radius $R$, centered at 0.

In order to prove Theorem 4.1, we also need to introduce the Banach space (cf.(3.4))

$$
\mathbb{H}:=\left\{(u, \psi, \eta) \in \mathcal{H}_{3,2}: \eta \in D(\mathbb{T})\right\}
$$

endowed with the norm

$$
\|(u, \psi, \eta)\|_{\mathbb{H}}^{2}=\|(u, \psi, \eta)\|_{\mathcal{H}_{3,2}}^{2}+\|\mathbb{T} \eta\|_{\mathcal{M}_{-1}}^{2}+\sup _{x \geq 1} x \int_{(x, \infty)} \nu(s)\|\eta(s)\|_{H^{-1}}^{2} d s .
$$

We recall that, while the embedding $\mathcal{H}_{3,2} \subset \mathcal{H}_{1,0}$ is only continuous, the space $\mathbb{H}$ is compactly embedded into $\mathcal{H}_{1,0}$ (see [45, Lemma 5.5]). Besides, closed balls of $\mathbb{H}$ are compact in $\mathcal{H}_{1,0}$ (see [6, Prop. 8.1]).

Theorem 4.1 follows from the existence of a bounded exponentially attracting set in $\mathbb{H}$, namely,

Theorem 4.4 Let the assumptions of Theorem 4.1 be satisfied. There exists $R_{1}>0$ and a closed ball $\mathcal{B}_{2}\left(R_{1}\right) \subset \mathbb{H} \cap \mathcal{H}_{1,0}^{M}$ which attracts $\mathcal{B}_{1,0}^{M}\left(R_{0}\right)$ (see Proposition 4.3) exponentially fast, that is,

$$
\operatorname{dist}_{\mathcal{H}_{1,0}}\left(S(t) \mathcal{B}_{1,0}^{M}\left(R_{0}\right), \mathcal{B}_{2}\left(R_{1}\right)\right) \leq C e^{-\rho(t-\tau)}, \forall t \geq \tau
$$

for some positive constants $C$ and $\rho$.

Proof. This proof is based on an argument similar to [25]. On account of Corollary 3.6, let us take $\Theta_{0}=\left(u_{0}, \psi_{0}, \eta_{0}\right) \in \mathcal{B}_{1,0}^{M}\left(R_{0}\right)$ and consider the corresponding trajectory $\left(u(t), \psi(t), \eta^{t}\right)=S(t)\left(u_{0}, \psi_{0}, \eta_{0}\right)$. From now on $c$ will denote a generic positive constant which is independent of time and which may vary even within the same line. Further dependencies of $c$ to other physical parameters will be pointed out as needed. In view of (N1), we can find positive constants $l_{1}, l_{2}$ so that

$$
f^{\prime}(y) \geq-l_{1}, \quad g^{\prime}(y) \geq-l_{2}, \quad \forall y \in \mathbb{R}
$$

Thus, in light of (4.20) and (N2'), it is not difficult to realize that we can also choose sufficiently large constants $\gamma_{1}>l_{1}$ and $\gamma_{2}>\beta+l_{2}$ with the following properties:

$$
\left\{\begin{array}{c}
\frac{1}{2}\|\nabla \zeta\|^{2}+\left(\gamma_{1}-2 l_{1}\right)\|\zeta\|^{2}-\left(f^{\prime}(u(t)) \zeta, \zeta\right) \geq 0 \\
\frac{1}{2}\left\|\nabla_{\Gamma} \zeta\right\|_{\Gamma}^{2}+\left(\gamma_{2}-2 l_{2}\right)\|\zeta\|_{\Gamma}^{2}-\left(g^{\prime}(\psi(t)) \zeta, \zeta\right)_{\Gamma} \geq 0
\end{array}\right.
$$


for all $\zeta \in \mathbb{V}_{1}$ and every $t \geq \tau$. Consequently, by setting $\tilde{f}(s)=f(s)+\gamma_{1} s, \widetilde{g}(s)=g(s)+\gamma_{2} s$, for all $s \in \mathbb{R}$, it is immediate that both $\widetilde{f}$ and $\widetilde{g}$ are monotone increasing and that they satisfy the same assumptions as the functions $f$ and $g$.

The following arguments are formal for the sake of simplicity, but they can be rigorously justified within the approximation scheme used in the proof of Theorem 3.3. We split the trajectory $\left(u(t), \psi(t), \eta^{t}\right)$ as follows (see [44], cf. also [25])

$$
\left(u(t), \psi(t), \eta^{t}\right)=\left(u_{d}(t), \psi_{d}(t), \eta_{d}^{t}\right)+\left(u_{c}(t), \psi_{c}(t), \eta_{c}^{t}\right)
$$

where

$$
\begin{aligned}
& \partial_{t} u_{d}+\int_{0}^{\infty} \nu(s) \eta_{d}^{t}(s) d s=0, \\
& \Upsilon\left(\mathbf{u}_{d}, \mathbf{v}\right)+\left(\widetilde{f}(u)-\widetilde{f}\left(u_{c}\right), v\right)+\left(\widetilde{g}(\psi)-\widetilde{g}\left(\psi_{c}\right), \varphi\right)_{\Gamma} \\
& +\left(\alpha \partial_{t} u_{d}, v\right)+\left(\partial_{t} \psi_{d}, \varphi\right)_{\Gamma}=\left(\mu_{d}, v\right)_{H^{-1}, H^{1}} \quad \forall \mathbf{v}=(v, \varphi) \in \mathbb{V}_{1}, \\
& \partial_{t} \eta_{d}^{t}-\mathbb{T} \eta_{d}^{t}=A_{N} \mu_{d}, \\
& u_{d}(\tau)=u_{0}, \quad \psi_{d}(\tau)=\psi_{0}, \quad \eta_{d}^{\tau}=\eta_{0},
\end{aligned}
$$

and

$$
\begin{aligned}
& \partial_{t} u_{c}+\int_{0}^{\infty} \nu(s) \eta_{c}^{t}(s) d s=0 \\
& \Upsilon\left(\mathbf{u}_{c}, \mathbf{v}\right)+\left(\widetilde{f}\left(u_{c}\right), v\right)+\left(\widetilde{g}\left(\psi_{c}\right), \varphi\right)_{\Gamma} \\
& +\left(\alpha \partial_{t} u_{c}, v\right)+\left(\partial_{t} \psi_{c}, \varphi\right)_{\Gamma}-\gamma_{1}(u, v)-\gamma_{2}(\psi, \varphi)_{\Gamma} \\
& =\left(\mu_{c}, v\right)_{H^{-1}, H^{1}}, \quad \forall \mathbf{v}=(v, \varphi) \in \mathbb{V}_{1}, \\
& \partial_{t} \eta_{c}^{t}-\mathbb{T} \eta_{c}^{t}=A_{N} \mu_{c}, \\
& u_{c}(\tau)=0, \quad \psi_{c}(\tau)=0, \quad \eta_{c}^{\tau}=0 .
\end{aligned}
$$

Let us first show that $\left(u_{d}(t), \psi_{d}(t), \eta_{d}^{t}\right)$ decays exponentially to zero with respect to the norm of $\mathcal{H}_{1,0}$. We begin by noting that, since $(u(t), \psi(t)) \in L^{\infty}\left((\tau, \infty) ; \mathbb{V}_{1}\right)$ and $\tilde{f}$ and $\widetilde{g}$ are non-decreasing, then we can easily adapt the proof of Lemma 4.2 , to find

$$
\left\|\left(u_{c}(t), \psi_{c}(t), \eta_{c}^{t}\right)\right\|_{\mathcal{H}_{1,0}}^{2}+\int_{\tau}^{\infty}\left(\alpha\left\|\partial_{t} u_{c}(y)\right\|^{2}+\left\|\partial_{t} \psi_{c}(y)\right\|_{\Gamma}^{2}\right) d y \leq c, \quad \forall t \geq \tau,
$$

which implies, on account of (4.1) and (4.22), that

$$
\left\|\left(u_{d}(t), \psi_{d}(t), \eta_{d}^{t}\right)\right\|_{\mathcal{H}_{1,0}}^{2}+\int_{\tau}^{\infty}\left(\alpha\left\|\partial_{t} u_{d}(y)\right\|^{2}+\left\|\partial_{t} \psi_{d}(y)\right\|_{\Gamma}^{2}\right) d y \leq c, \quad \forall t \geq \tau
$$

where $c$ is obviously independent of $\alpha$. 
We now take the scalar product of equation (4.23) with $\mu_{d}(t)$ in $L^{2}(\Omega)$, and the scalar product of equation (4.25) with $A_{N}^{-1} \eta_{d}^{t}$ in $\mathcal{M}_{0}$. Also, we take $\mathbf{v}=\left(\partial_{t} u_{d}(t), \partial_{t} \psi_{d}(t)\right)$ in (4.24). Adding together the resulting relationships, we find

$$
\begin{aligned}
& \frac{1}{2} \frac{d}{d t}\left[\left\|\nabla u_{d}\right\|^{2}+\left\|\nabla_{\Gamma} \psi_{d}\right\|_{\Gamma}^{2}+\beta\left\|\psi_{d}\right\|_{\Gamma}^{2}+\left\|\eta_{d}^{t}\right\|_{\mathcal{M}_{-1}}^{2}\right] \\
& +\frac{d}{d t}\left[\left(\widetilde{f}(u)-\widetilde{f}\left(u_{c}\right), u_{d}\right)+\left(\widetilde{g}(\psi)-\widetilde{g}\left(\psi_{c}\right), \psi_{d}\right)_{\Gamma}\right] \\
& +\alpha\left\|\partial_{t} u_{d}\right\|^{2}+\left\|\partial_{t} \psi_{d}\right\|_{\Gamma}^{2}-\int_{\tau}^{\infty} \nu^{\prime}(s)\left\|\eta_{d}^{t}(s)\right\|_{H^{-1}}^{2} d s \\
& =\left(\widetilde{f}^{\prime}(u) \partial_{t} u-\widetilde{f}^{\prime}\left(u_{c}\right) \partial_{t} u_{c}, u_{d}\right)+\left(\widetilde{g}^{\prime}(\psi) \partial_{t} \psi-\widetilde{g}^{\prime}\left(\psi_{c}\right) \partial_{t} \psi_{c}, \psi_{d}\right)_{\Gamma} .
\end{aligned}
$$

Let us consider the functional

$$
\begin{aligned}
E_{d}(t) & =\left\|\nabla u_{d}(t)\right\|^{2}+\left\|\nabla_{\Gamma} \psi_{d}(t)\right\|_{\Gamma}^{2}+\beta\left\|\psi_{d}(t)\right\|_{\Gamma}^{2}+\left\|\eta_{d}^{t}(t)\right\|_{\mathcal{M}_{-1}}^{2} \\
& +\left(\widetilde{f}(u(t))-\widetilde{f}\left(u_{c}(t)\right), u_{d}(t)\right)-\left(\widetilde{f}^{\prime}(u(t)) u_{d}(t), u_{d}(t)\right) \\
& +\left(\widetilde{g}(\psi(t))-\widetilde{g}\left(\psi_{c}(t)\right), \psi_{d}(t)\right)_{\Gamma}-\left(\widetilde{g}^{\prime}(\psi(t)) \psi_{d}(t), \psi_{d}(t)\right)_{\Gamma}
\end{aligned}
$$

and note that, on account of (4.20) and (4.21), we can find a positive constant $C_{1}$, independent of $t$ and $\alpha$, such that, for all $t \geq \tau$,

$$
C_{1}^{-1}\left\|\left(u_{d}(t), \psi_{d}(t), \eta_{d}^{t}\right)\right\|_{\mathcal{H}_{1,0}}^{2} \leq E_{d}(t) \leq C_{1}\left\|\left(u_{d}(t), \psi_{d}(t), \eta_{d}^{t}\right)\right\|_{\mathcal{H}_{1,0}}^{2} .
$$

Indeed, (4.21) yields

$$
\left(\tilde{f}(u)-\widetilde{f}\left(u_{c}\right), u_{d}\right)-\left(\widetilde{f}^{\prime}(u) u_{d}, u_{d}\right) \geq\left(\gamma_{1}-2 l_{1}\right)\left\|u_{d}\right\|^{2}-\left(f^{\prime}(u) u_{d}, u_{d}\right) \geq-\frac{1}{2}\left\|\nabla u_{d}\right\|^{2},
$$

and a similar inequality holds for the last two terms on the right-hand side of (4.34). Then, recalling (K5) and performing simple computations, we can rewrite (4.33) as follows:

$$
\frac{1}{2} \frac{d}{d t} E_{d}(t)+\frac{\lambda}{2}\left\|\eta_{d}^{t}\right\|_{\mathcal{M}_{-1}}^{2}-\frac{1}{2} \int_{0}^{\infty} \nu^{\prime}(s)\left\|\eta^{t}(s)\right\|_{H^{-1}}^{2} d s \leq \Lambda_{d}(t), \quad \forall t \geq \tau
$$

where

$$
\begin{aligned}
\Lambda_{d} & =\left(\left(\widetilde{f}^{\prime}(u)-\widetilde{f}^{\prime}\left(u_{c}\right)\right) \partial_{t} u_{c}, u_{d}\right)+\left(\left(\widetilde{g}^{\prime}(\psi)-\widetilde{g}^{\prime}\left(\psi_{c}\right)\right) \partial_{t} \psi_{c}, \psi_{d}\right)_{\Gamma} \\
& -\left(\widetilde{f}^{\prime \prime}(u) \partial_{t} u,\left(u_{d}\right)^{2}\right)-\left(\widetilde{g}^{\prime \prime}(\psi) \partial_{t} \psi,\left(\psi_{d}\right)^{2}\right)_{\Gamma} .
\end{aligned}
$$

Following the proof of Lemma 4.2, we set $\bar{u}_{d}=u_{d}-\left\langle u_{d}\right\rangle$ and $\bar{\psi}_{d}=\psi_{d}-\left\langle u_{d}\right\rangle$ (note that $\left.\left\langle u_{d}(t)\right\rangle=\langle u(t)\rangle\right)$. Then we introduce the auxiliary functional

$$
I_{d}(t)=-\int_{0}^{\infty} \nu(s)\left(\bar{u}_{d}(t), A_{N}^{-1} \eta_{d}^{t}(s)\right) d s,
$$


and we observe that, using equations (4.23)-(4.24), we have (see also (4.3))

$$
\begin{aligned}
\frac{d}{d t} I_{d}(t) & =\left\|\partial_{t} u_{d}(t)\right\|_{H^{-1}}^{2}-\int_{0}^{\infty} \nu^{\prime}(s)\left(\bar{u}_{d}(t), A_{N}^{-1} \eta_{d}^{t}(s)\right) d s-\left(\bar{u}_{d}(t), \mu_{d}(t)\right) \\
& =\left\|\partial_{t} u_{d}(t)\right\|_{H^{-1}}^{2}-\int_{0}^{\infty} \nu^{\prime}(s)\left(\bar{u}_{d}(t), A_{N}^{-1} \eta_{d}^{t}(s)\right) d s-\left\|\nabla \bar{u}_{d}(t)\right\|^{2} \\
& -\left\|\nabla_{\Gamma} \bar{\psi}_{d}(t)\right\|_{\Gamma}^{2}-\beta\left(\psi_{d}(t), \bar{\psi}_{d}(t)\right)_{\Gamma}-\left(\widetilde{f}(u(t))-\tilde{f}\left(u_{c}(t)\right), \bar{u}_{d}(t)\right) \\
& -\left(\widetilde{g}(\psi(t))-\widetilde{g}\left(\psi_{c}(t)\right), \bar{\psi}_{d}(t)\right)_{\Gamma}-\frac{1}{2} \frac{d}{d t}\left[\alpha\left\|\bar{u}_{d}(t)\right\|^{2}+\left\|\bar{\psi}_{d}(t)\right\|_{\Gamma}^{2}\right] .
\end{aligned}
$$

Therefore, collecting (4.36) and (4.38), we find

$$
\begin{aligned}
& \frac{1}{2} \frac{d}{d t}\left[\widetilde{E}_{d}(t)+2 \varepsilon I_{d}(t)\right]+\frac{\lambda}{2}\left\|\eta_{d}^{t}\right\|_{\mathcal{M}_{-1}}^{2}-\frac{1}{2} \int_{0}^{\infty} \nu^{\prime}(s)\left\|\eta^{t}(s)\right\|_{H^{-1}}^{2} d s \\
& -\varepsilon\left\|\partial_{t} u_{d}(t)\right\|_{H^{-1}}^{2}+\varepsilon \int_{0}^{\infty} \nu^{\prime}(s)\left(\bar{u}_{d}(t), A_{N}^{-1} \eta_{d}^{t}(s)\right) d s \\
& +\varepsilon\left(\left\|\nabla \bar{u}_{d}(t)\right\|^{2}+\left\|\nabla_{\Gamma} \psi_{d}(t)\right\|_{\Gamma}^{2}+\beta\left\|\psi_{d}(t)\right\|_{\Gamma}^{2}\right) \\
& +\varepsilon\left(\widetilde{f}(u(t))-\widetilde{f}\left(u_{c}(t)\right), u_{d}(t)\right)+\varepsilon\left(\widetilde{g}(\psi(t))-\widetilde{g}\left(\psi_{c}(t)\right), \psi_{d}(t)\right)_{\Gamma} \\
& \leq \Lambda_{d}(t)+\varepsilon\left(\tilde{f}(u(t))-\widetilde{f}\left(u_{c}(t)\right), 1\right)\left\langle u_{d}(t)\right\rangle+\varepsilon\left\langle u_{d}(t)\right\rangle\left(\widetilde{g}(\psi(t))-\widetilde{g}\left(\psi_{c}(t)\right), 1\right)_{\Gamma} \\
& +\varepsilon \beta\left\langle u_{d}(t)\right\rangle\left(1, \psi_{d}(t)\right)_{\Gamma},
\end{aligned}
$$

where we have set

$$
\widetilde{E}_{d}(t)=E_{d}(t)+\varepsilon\left(\alpha\left\|\bar{u}_{d}(t)\right\|^{2}+\left\|\bar{\psi}_{d}(t)\right\|_{\Gamma}^{2}\right) .
$$

Here $\varepsilon \in(0, \lambda)$ is a parameter which will be properly chosen. On account of (4.37), from (4.35), for $\varepsilon>0$ small enough, we can easily find a positive constant $C_{2}=C_{2}(\varepsilon, M)$ such that, for all $t \geq \tau$,

$$
C_{2}^{-1}\left\|\left(u_{d}(t), \psi_{d}(t), \eta_{d}^{t}\right)\right\|_{\mathcal{H}_{1,0}}^{2} \leq \widetilde{E}_{d}(t)+2 \varepsilon I_{d}(t) \leq C_{2}\left\|\left(u_{d}(t), \psi_{d}(t), \eta_{d}^{t}\right)\right\|_{\mathcal{H}_{1,0}}^{2} .
$$

Let us now the right-hand side of (4.39)). On account of (N2'), (4.20) and estimates (4.31)(4.32), (4.1), from Hölder and Young inequalities, it follows that

$$
\begin{aligned}
\Lambda_{d}(t) & \leq c\left\|\partial_{t} u(t)\right\|\left\|u_{d}(t)\right\|_{H^{1}}^{2}+c\left\|\partial_{t} \psi(t)\right\|_{\Gamma}\left\|\psi_{d}(t)\right\|_{H^{1}(\Gamma)}^{2} \\
& \leq c\left(\left\|\partial_{t} u(t)\right\|+\left\|\partial_{t} \psi(t)\right\|_{\Gamma}\right)\left(\left\|\nabla u_{d}(t)\right\|^{2}+\left\|\nabla_{\Gamma} \psi_{d}(t)\right\|_{\Gamma}^{2}+\beta\left\|\psi_{d}(t)\right\|_{\Gamma}^{2}\right) \\
& \leq c\left(\left\|\partial_{t} u(t)\right\|+\left\|\partial_{t} \psi(t)\right\|_{\Gamma}\right) \widetilde{E}_{d}(t), \quad \forall t \geq \tau .
\end{aligned}
$$

Moreover, we have that

$$
\begin{aligned}
& \varepsilon\left(\tilde{f}(u(t))-\widetilde{f}\left(u_{c}(t)\right), 1\right)\left\langle u_{d}(t)\right\rangle+\varepsilon \beta\left\langle u_{d}(t)\right\rangle\left(1, \psi_{d}(t)\right)_{\Gamma} \\
& +\varepsilon\left\langle u_{d}(t)\right\rangle\left(\widetilde{g}(\psi(t))-\widetilde{g}\left(\psi_{c}(t)\right), 1\right)_{\Gamma} \\
& \leq c\left(\left\|\bar{u}_{d}\right\|^{2}+\left\|\bar{\psi}_{d}\right\|_{\Gamma}^{2}\right) \leq c \widetilde{E}_{d}(t), \quad \forall t \geq \tau .
\end{aligned}
$$


On the other hand, arguing as in (4.12), we find

$$
\begin{aligned}
& \varepsilon\left\|\partial_{t} u_{d}(t)\right\|_{H^{-1}}^{2} \leq \varepsilon\left\|\eta_{d}^{t}\right\|_{\mathcal{M}_{-1}}^{2}, \\
& -\varepsilon \int_{0}^{\infty} \nu^{\prime}(s)\left(\bar{u}_{d}(t), A_{N}^{-1} \eta_{d}^{t}(s)\right) d s \leq \frac{\varepsilon}{2}\left\|\nabla \bar{u}_{d}(t)\right\|^{2}-2 \varepsilon C_{\Omega}^{4} \nu_{0} \int_{0}^{\infty} \nu^{\prime}(s)\left\|\eta_{d}^{t}(s)\right\|_{H^{-1}}^{2} d s .
\end{aligned}
$$

Therefore, using the above inequalities (4.42)-(4.45), from (4.39) we infer

$$
\begin{aligned}
& \frac{1}{2} \frac{d}{d t}\left[\widetilde{E}_{d}(t)+2 \varepsilon I_{d}(t)\right]+\left(\frac{\lambda}{2}-\varepsilon\right)\left\|\eta_{d}^{t}\right\|_{\mathcal{M}_{-1}}^{2} \\
& -\left(\frac{1}{2}-2 \varepsilon C_{\Omega}^{4} \nu_{0}\right) \int_{0}^{\infty} \nu^{\prime}(s)\left\|\eta_{d}^{t}(s)\right\|_{H^{-1}}^{2} d s \\
& +\varepsilon\left(\frac{1}{2}\left\|\nabla \bar{u}_{d}(t)\right\|^{2}+\left\|\nabla_{\Gamma} \psi_{d}(t)\right\|_{\Gamma}^{2}+\beta\left\|\psi_{d}(t)\right\|_{\Gamma}^{2}\right) \\
& +\varepsilon\left(\widetilde{f}(u(t))-\widetilde{f}\left(u_{c}(t)\right), u_{d}(t)\right)+\varepsilon\left(\widetilde{g}(\psi(t))-\widetilde{g}\left(\psi_{c}(t)\right), \psi_{d}(t)\right)_{\Gamma} \\
& \leq c\left(1+\left\|\partial_{t} u(t)\right\|+\left\|\partial_{t} \psi(t)\right\|_{\Gamma}\right) \widetilde{E}_{d}(t),
\end{aligned}
$$

for all $t \geq \tau$. Thus, setting $\widehat{E}_{d}(t)=\widetilde{E}_{d}(t)+2 \varepsilon I_{d}(t)$ and choosing $\varepsilon>0$ sufficiently small, and a positive constant $C_{\varepsilon} \in(0, \varepsilon)$, from (4.46) we deduce that

$$
\frac{d}{d t} \widehat{E}_{d}(t)+C_{\varepsilon} \widehat{E}_{d}(t) \leq c\left(1+\left\|\partial_{t} u(t)\right\|+\left\|\partial_{t} \psi(t)\right\|_{\Gamma}\right) \widehat{E}_{d}(t)
$$

It is left to notice that, owing to (4.1), we get

$$
\int_{\tau}^{t}\left(1+\left\|\partial_{t} u(y)\right\|+\left\|\partial_{t} \psi(y)\right\|_{\Gamma}\right) d y \leq c(t-\tau)+\frac{c}{\alpha}, \quad \forall t \geq \tau,
$$

where $c$ is independent of $\alpha$. Bound (4.48) allows us to apply a suitable Gronwall's inequality (see, e.g., [7, Lemma 3.5]) which yields

$$
\widehat{E}_{d}(t) \leq c e^{-C_{\varepsilon}(t-\tau)}, \quad \forall t \geq \tau
$$

Thus, recalling (4.41), (4.49) gives the desired exponential decay of $\left\|\left(u_{d}(t), \psi_{d}(t), \eta_{d}^{t}\right)\right\|_{\mathcal{H}_{1,0}}$.

Let us now obtain a bound for $\left\|\left(u_{c}(t), \psi_{c}(t), \eta_{c}^{t}\right)\right\|_{\mathbb{H}}$. To this end, we argue as in the proof of Theorem 3.4, namely, we set

$$
\widetilde{u}_{c}=\partial_{t} u_{c}, \quad \widetilde{\psi}_{c}=\partial_{t} \psi_{c}, \quad \widetilde{\eta}_{c}=\partial_{t} \eta_{c}
$$


and observe that $\left(\widetilde{u}_{c}, \widetilde{\psi}_{c}, \widetilde{\eta}_{c}\right)$ solves (cf. (4.27)-(4.30))

$$
\begin{aligned}
& \partial_{t} \widetilde{u}_{c}+\int_{0}^{\infty} \nu(s) \widetilde{\eta}_{c}^{t}(s) d s=0, \\
& \Upsilon\left(\widetilde{\mathbf{u}}_{c}, \mathbf{v}\right)+\left(\widetilde{f}^{\prime}\left(u_{c}\right) \widetilde{u}_{c}, v\right)+\left(\widetilde{g}^{\prime}\left(\psi_{c}\right) \widetilde{\psi}_{c}, \varphi\right)_{\Gamma} \\
& +\left(\alpha \partial_{t} \widetilde{u}_{c}, v\right)+\left(\partial_{t} \widetilde{\psi}_{c}, \varphi\right)_{\Gamma}-\gamma_{1}\left(\partial_{t} u, v\right)-\gamma_{2}\left(\partial_{t} \psi, \varphi\right)_{\Gamma} \\
& =\left(\partial_{t} \mu_{c}, v\right)_{H^{-1}, H^{1}}, \quad \forall \mathbf{v}=(v, \varphi) \in \mathbb{V}_{1}, \\
& \partial_{t} \widetilde{\eta}_{c}^{t}-\mathbb{T} \widetilde{\eta}_{c}^{t}=A_{N}\left(\partial_{t} \mu_{c}\right), \\
& \widetilde{u}_{c}(\tau)=0, \quad \widetilde{\psi}_{c}(\tau)=0, \quad \widetilde{\eta}_{c}^{\tau}=0 .
\end{aligned}
$$

The analogue of (4.33) for (4.50)-(4.53) is the following identity

$$
\frac{1}{2} \frac{d}{d t} E_{c}(t)-\int_{0}^{\infty} \nu^{\prime}(s)\left\|\widetilde{\eta}_{c}^{t}(s)\right\|_{H^{-1}}^{2} d s+\alpha\left\|\partial_{t} \widetilde{u}_{c}\right\|^{2}+\left\|\partial_{t} \widetilde{\psi}_{c}\right\|_{\Gamma}^{2}=\Lambda_{c}(t)
$$

where

$$
\begin{aligned}
E_{c}(t) & =\left\|\nabla \widetilde{u}_{c}(t)\right\|^{2}+\left\|\nabla_{\Gamma} \widetilde{\psi}_{c}(t)\right\|_{\Gamma}^{2}+\beta\left\|\widetilde{\psi}_{c}(t)\right\|_{\Gamma}^{2}+\left\|\widetilde{\eta}_{c}^{t}\right\|_{\mathcal{M}_{-1}}^{2} \\
& +\left(\widetilde{f}^{\prime}\left(u_{c}(t)\right) \widetilde{u}_{c}(t), \widetilde{u}_{c}(t)\right)+\left(\widetilde{g}^{\prime}\left(u_{c}(t)\right) \widetilde{\psi}_{c}(t), \widetilde{\psi}_{c}(t)\right)_{\Gamma}
\end{aligned}
$$

and

$$
\begin{aligned}
\Lambda_{c}(t) & =\frac{1}{2}\left(\widetilde{f}^{\prime \prime}\left(u_{c}(t)\right) \partial_{t} u_{c}(t),\left(\widetilde{u}_{c}(t)\right)^{2}\right)+\frac{1}{2}\left(\widetilde{g}^{\prime \prime}\left(\psi_{c}(t)\right) \partial_{t} \psi_{c}(t),\left(\widetilde{\psi}_{c}(t)\right)^{2}\right)_{\Gamma} \\
& +\gamma_{1}\left(\partial_{t} u(t), \partial_{t} \widetilde{u}_{c}(t)\right)+\gamma_{2}\left(\partial_{t} \psi(t), \partial_{t} \widetilde{\psi}_{c}(t)\right)_{\Gamma} .
\end{aligned}
$$

Note that, using $(\mathrm{N} 2$ '), (4.20), the fact that $\tilde{f}, \widetilde{g}$ are monotone increasing and employing estimate (4.31), we can find a positive constant $C_{3}$ such that

$$
C_{3}^{-1}\left\|\left(\widetilde{u}_{c}(t), \widetilde{\psi}_{c}(t), \widetilde{\eta}_{c}^{t}\right)\right\|_{\mathcal{H}_{1,0}}^{2} \leq E_{c}(t) \leq C_{3}\left\|\left(\widetilde{u}_{c}(t), \widetilde{\psi}_{c}(t), \widetilde{\eta}_{c}^{t}\right)\right\|_{\mathcal{H}_{1,0}}^{2}, \quad \forall t \geq \tau
$$

Similarly to (4.37), we introduce the functional

$$
I_{c}(t)=-\int_{0}^{\infty} \nu(s)\left(\widetilde{u}_{c}(t), A_{N}^{-1} \widetilde{\eta}_{c}^{t}(s)\right) d s
$$


and observe that, using equations (4.50)-(4.51), we obtain $\left(\left\langle u_{c}(t)\right\rangle=0\right.$, for all $\left.t \geq \tau\right)$ :

$$
\begin{aligned}
\frac{d}{d t} I_{c}(t) & =\left\|\partial_{t} \widetilde{u}_{c}(t)\right\|_{H^{-1}}^{2}-\int_{0}^{\infty} \nu^{\prime}(s)\left(\widetilde{u}_{c}(t), A_{N}^{-1} \widetilde{\eta}_{c}^{t}(s)\right) d s-\left(\widetilde{u}_{c}(t), \partial_{t} \mu_{c}(t)\right) \\
& =\left\|\partial_{t} \widetilde{u}_{c}(t)\right\|_{H^{-1}}^{2}-\int_{0}^{\infty} \nu^{\prime}(s)\left(\widetilde{u}_{c}(t), A_{N}^{-1} \widetilde{\eta}_{c}^{t}(s)\right) d s-\left\|\nabla \widetilde{u}_{c}(t)\right\|^{2} \\
& -\left\|\nabla_{\Gamma} \widetilde{\psi}_{c}(t)\right\|_{\Gamma}^{2}-\beta\left\|\widetilde{\psi}_{c}(t)\right\|_{\Gamma}^{2}-\left(\widetilde{f}^{\prime}\left(u_{c}(t)\right) \widetilde{u}_{c}(t), \widetilde{u}_{c}(t)\right) \\
& -\left(\widetilde{g}^{\prime}\left(\psi_{c}(t)\right) \widetilde{\psi}_{c}(t), \widetilde{\psi}_{c}(t)\right)_{\Gamma}-\frac{1}{2} \frac{d}{d t}\left[\alpha\left\|\widetilde{u}_{c}(t)\right\|^{2}+\left\|\widetilde{\psi}_{c}(t)\right\|_{\Gamma}^{2}\right] \\
& -\gamma_{1}\left(\partial_{t} u(t), \widetilde{u}_{c}(t)\right)-\gamma_{2}\left(\partial_{t} \psi(t), \widetilde{\psi}_{c}(t)\right)_{\Gamma} .
\end{aligned}
$$

Setting

$$
\widetilde{E}_{c}(t)=E_{c}(t)+\alpha\left\|\widetilde{u}_{c}(t)\right\|^{2}+\left\|\widetilde{\psi}_{c}(t)\right\|_{\Gamma}^{2}+2 \varepsilon I_{c}(t)
$$

and arguing as to getting (4.39), we deduce

$$
\begin{aligned}
& \frac{1}{2} \frac{d}{d t} \widetilde{E}_{c}(t)-\int_{0}^{\infty} \nu^{\prime}(s)\left\|\widetilde{\eta}_{c}^{t}(s)\right\|_{H^{-1}}^{2} d s+\alpha\left\|\partial_{t} \widetilde{u}_{c}(t)\right\|^{2}+\left\|\partial_{t} \widetilde{\psi}_{c}(t)\right\|_{\Gamma}^{2}-\varepsilon\left\|\partial_{t} \widetilde{u}_{c}(t)\right\|_{H^{-1}}^{2} \\
& +\varepsilon \int_{0}^{\infty} \nu^{\prime}(s)\left(\widetilde{u}_{c}(t), A_{N}^{-1} \widetilde{\eta}_{c}^{t}(s)\right) d s+\varepsilon\left(\left\|\nabla \widetilde{u}_{c}(t)\right\|^{2}+\left\|\nabla_{\Gamma} \widetilde{\psi}_{c}(t)\right\|_{\Gamma}^{2}+\beta\left\|\widetilde{\psi}_{c}(t)\right\|_{\Gamma}^{2}\right) \\
& +\varepsilon\left(\widetilde{f}^{\prime}\left(u_{c}(t)\right) \widetilde{u}_{c}(t), \widetilde{u}_{c}(t)\right)+\varepsilon\left(\widetilde{g}^{\prime}\left(\psi_{c}(t)\right) \widetilde{\psi}_{c}(t), \widetilde{\psi}_{c}(t)\right)_{\Gamma} \\
& =\Lambda_{c}(t)-\gamma_{1} \varepsilon\left(\partial_{t} u(t), \widetilde{u}_{c}(t)\right)-\gamma_{2} \varepsilon\left(\partial_{t} \psi(t), \widetilde{\psi}_{c}(t)\right)_{\Gamma} .
\end{aligned}
$$

Hence, recalling (N2'), using Hölder and Young inequalities and exploiting (4.1) and (4.31), we estimate the terms on the right-hand side of (4.58) as follows:

$$
\begin{aligned}
& \Lambda_{c}-\gamma_{1} \varepsilon\left(\partial_{t} u, \widetilde{u}_{c}\right)-\gamma_{2} \varepsilon\left(\partial_{t} \psi, \widetilde{\psi}_{c}\right)_{\Gamma} \\
& \leq \frac{1}{2}\left(\alpha\left\|\partial_{t} \widetilde{u}_{c}\right\|^{2}+\left\|\partial_{t} \widetilde{\psi}_{c}\right\|_{\Gamma}^{2}\right)+c\left(\left\|\partial_{t} u\right\|^{2}+\left\|\partial_{t} \psi\right\|_{\Gamma}^{2}\right) \\
& +c\left[\left(\left\|\partial_{t} u_{c}\right\|+1\right)\left\|\widetilde{u}_{c}\right\|_{H^{1}}^{2}+\left(\left\|\partial_{t} \psi_{c}\right\|_{\Gamma}+1\right)\left\|\widetilde{\psi}_{c}\right\|_{H^{1}(\Gamma)}^{2}\right] .
\end{aligned}
$$

Therefore, on account of (4.59), we can argue as to getting (4.47) and we deduce

$$
\begin{aligned}
\frac{d}{d t} \widetilde{E}_{c}(t)+C_{\varepsilon} \widetilde{E}_{c}(t) & \leq c\left(\left\|\partial_{t} u(t)\right\|^{2}+\left\|\partial_{t} \psi(t)\right\|_{\Gamma}^{2}\right) \\
& +c\left(1+\left\|\partial_{t} u_{c}(t)\right\|+\left\|\partial_{t} \psi_{c}(t)\right\|_{\Gamma}\right) \widetilde{E}_{c}(t),
\end{aligned}
$$

for all $t \geq \tau$, where $C_{\varepsilon}>0$ is independent of $\alpha$. Hence, integrating (4.60) with respect to time on the interval $(\tau, t)$, noting that $E_{c}(\tau)=0$ and exploiting (4.1), (4.31) and (4.55), we obtain

$$
\left\|\left(\widetilde{u}_{c}(t), \widetilde{\psi}_{c}(t), \widetilde{\eta}_{c}^{t}\right)\right\|_{\mathcal{H}_{1,0}}^{2} \leq c, \quad \forall t \geq \tau
$$


where $c$ depends on $\alpha$.

Observe now that, using equation (4.27), we have

$$
\left\|\eta_{c}^{t}\right\|_{\mathcal{M}_{1}}^{2}=-\int_{0}^{\infty} \nu(s)\left[\left(\nabla \partial_{t} u_{c}, \nabla \eta_{c}^{t}(s)\right)+\left(\partial_{t} u_{c}, \eta_{c}^{t}(s)\right)\right] d s,
$$

which entails, thanks to (4.61) and Young's inequality, that

$$
\left\|\eta_{c}^{t}\right\|_{\mathcal{M}_{1}}^{2} \leq c, \quad \forall t \geq \tau
$$

Let us now consider equation (4.29) and set $\bar{\mu}_{c}(t)=\mu_{c}(t)-\left\langle\mu_{c}(t)\right\rangle$. Multiplying (4.29) by $\bar{\mu}_{c}(t)$, taking the scalar product in $\mathcal{M}_{0}$, recalling (K3)-(K4) and exploiting estimate (4.61), we deduce

$$
\begin{aligned}
\left\|\nabla \mu_{c}(t)\right\|^{2} & =\int_{0}^{\infty} \nu(s)\left(\bar{\mu}_{c}(t), \partial_{t} \eta_{c}^{t}(s)\right) d s-\int_{0}^{\infty} \nu^{\prime}(s)\left(\bar{\mu}_{c}(t), \eta_{c}^{t}(s)\right) d s \\
& \leq c_{\varepsilon}\left(\left\|\partial_{t} \eta_{c}^{t}\right\|_{\mathcal{M}_{-1}}^{2}-\int_{0}^{\infty} \nu^{\prime}(s)\left\|\eta_{c}^{t}(s)\right\|_{H^{-1}}^{2} d s\right)+\varepsilon\left\|\nabla \mu_{c}(t)\right\|^{2} \\
& \leq c_{\varepsilon}\left(1-\int_{0}^{\infty} \nu^{\prime}(s)\left\|\eta_{c}^{t}(s)\right\|_{H^{-1}}^{2} d s\right)+\varepsilon\left\|\nabla \mu_{c}(t)\right\|^{2},
\end{aligned}
$$

for every $\varepsilon>0$, where $c_{\varepsilon}$ is a sufficiently large constant that depends on $\varepsilon$ and $\alpha$. Moreover, multiplying (4.29) by $A_{N}^{-1} \eta_{c}^{t}$ and taking the scalar product in $\mathcal{M}_{0}$, we find (note that $\left\langle\eta_{c}^{t}\right\rangle=0$ )

$$
\begin{aligned}
-\int_{0}^{\infty} \nu^{\prime}(s)\left\|\eta_{c}^{t}(s)\right\|_{H^{-1}}^{2} d s & =-\int_{0}^{\infty} \nu(s)\left(A_{N}^{-1 / 2} \partial_{t} \eta_{c}^{t}(s), A_{N}^{-1 / 2} \eta_{c}^{t}(s)\right) d s \\
& +\int_{0}^{\infty} \nu(s)\left(\eta_{c}^{t}(s), \bar{\mu}_{c}(t)\right) d s,
\end{aligned}
$$

which yields, using the Poincaré-Wirtinger inequality and (4.31), (4.61),

$$
-\int_{0}^{\infty} \nu^{\prime}(s)\left\|\eta_{c}^{t}(s)\right\|_{H^{-1}}^{2} d s \leq c_{\varepsilon}+\varepsilon\left\|\nabla \mu_{c}(t)\right\|^{2},
$$

for every $\varepsilon>0$. Therefore, combining (4.64) with (4.65) and choosing $\varepsilon$ sufficiently small, it is readily seen that

$$
\left\|\nabla \mu_{c}(t)\right\|^{2} \leq c, \quad \forall t \geq \tau .
$$

On the other hand, from equation (4.28), we have

$$
\begin{aligned}
\left\langle\mu_{c}(t)\right\rangle & =|\Omega|^{-1}\left[\beta\left(1, \psi_{c}(t)\right)+\left(\widetilde{f}\left(u_{c}(t)\right), 1\right)+\left(\widetilde{g}\left(\psi_{c}(t)\right), 1\right)_{\Gamma}+\left(\partial_{t} \psi_{c}(t), 1\right)_{\Gamma}\right] \\
& -\gamma_{1}\langle u(t)\rangle-\gamma_{2}|\Omega|^{-1}(\psi(t), 1)_{\Gamma} .
\end{aligned}
$$

Thus, in view of (N2'), thanks to (4.1), (4.31), (4.61), we get

$$
\left\langle\mu_{c}(t)\right\rangle \leq c, \quad \forall t \geq \tau .
$$


Furthermore, on account of the representation formula (see [30], cf. also [7, A.1]), we have

$$
-\mathbb{T} \eta_{c}^{t}(s)=\left\{\begin{array}{c}
A_{N} \mu(t-s), \quad 0<s \leq t-\tau \\
0, \quad s>t-\tau
\end{array}\right.
$$

Thus, owing to (4.66) and (4.68), we get

$$
\left\|\mathbb{T} \eta_{c}^{t}\right\|_{\mathcal{M}_{-1}}^{2} \leq c, \quad \forall t \geq \tau
$$

The tail control follows once more from the representation formula, thanks to (K5) and (4.66) (see, e.g., [8, Lemma 3.4]). More precisely, we have

$$
\sup _{x \geq 1} x \int_{(x, \infty)} \nu(s)\left\|\eta_{c}^{t}(s)\right\|_{H^{-1}}^{2} d s \leq c, \quad \forall t \geq \tau .
$$

We are left to prove uniform bounds for $\left(u_{c}, \psi_{c}\right)$ in the $\mathbb{V}_{3}$-norm. Recalling, for instance, the end of the proof of [2, Theorem 2.3], we have that $\left(u_{c}, \psi_{c}\right) \in L_{l o c}^{2}\left((\tau, \infty) ; \mathbb{V}_{2}\right)$ and satisfies the following elliptic boundary value problem

$$
\begin{aligned}
& -\Delta u_{c}(t)=h_{c}^{1}(t), \quad \text { a.e. in } \Omega \times(\tau, \infty), \\
& -\Delta_{\Gamma} \psi_{c}(t)+\partial_{\mathbf{n}} u_{c}(t)+\beta \psi_{c}(t)=h_{c}^{2}(t), \quad \text { a.e. on } \Gamma \times(\tau, \infty),
\end{aligned}
$$

where

$$
h_{c}^{1}:=-\alpha \partial_{t} u_{c}-\widetilde{f}\left(u_{c}\right)+\mu_{c}-\gamma_{1} u, \quad h_{c}^{2}:=-\partial_{t} \psi_{c}-\widetilde{g}\left(\psi_{c}\right)-\gamma_{2} \psi .
$$

On account of (4.1), (4.31), (4.61), (4.66), (4.68) and (N2'), we have that

$$
\left\|h_{c}^{1}(t)\right\|+\left\|h_{c}^{2}(t)\right\|_{\Gamma} \leq c, \quad \forall t \geq \tau
$$

where $c$ depends on $\alpha$. Thus, using (4.73) an application of [39, Lemma A.1] yields

$$
\left\|\left(u_{c}(t), \psi_{c}(t)\right)\right\|_{\mathbb{V}_{2}} \leq c\left(\left\|h_{c}^{1}(t)\right\|+\left\|h_{c}^{2}(t)\right\|_{\Gamma}\right) \leq c, \quad \forall t \geq \tau .
$$

Exploiting now (4.74), using once more (4.1), (4.31), (4.61), (4.66), (4.68) and recalling the embeddings $H^{2}(\Omega) \hookrightarrow L^{\infty}(\Omega), H^{2}(\Gamma) \hookrightarrow L^{\infty}(\Gamma)$, it not difficult to realize that

$$
\left\|h_{c}^{1}(t)\right\|_{H^{1}}+\left\|h_{c}^{2}(t)\right\|_{H^{1}(\Gamma)} \leq c, \quad \forall t \geq \tau .
$$

Thus, a further application of [39, Lemma A.1] to (4.71)-(4.72) gives

$$
\left\|\left(u_{c}(t), \psi_{c}(t)\right)\right\|_{\mathbb{V}_{3}} \leq c\left(\left\|h_{c}^{1}(t)\right\|_{H^{1}}+\left\|h_{c}^{2}(t)\right\|_{H^{1}(\Gamma)}\right) \leq c, \forall t \geq \tau .
$$

Collecting (4.61), (4.63), (4.69), (4.70) and (4.75), we eventually get

$$
\left\|\left(u_{c}(t), \psi_{c}(t), \eta_{c}^{t}\right)\right\|_{\mathbb{H}} \leq c, \quad \forall t \geq \tau,
$$

and the proof follows. 
We conclude this section observing that $\left(\mathcal{H}_{1,0}^{M}, S(t)\right)$ is a gradient system, namely, it has a Lyapunov functional $\mathcal{L}: \mathcal{H}_{1,0}^{M} \rightarrow \mathbb{R}$ defined by (cf. (3.26))

$$
\mathcal{L}(u, \psi, \eta):=\|\nabla u\|^{2}+\left\|\nabla_{\Gamma} \psi\right\|_{\Gamma}^{2}+\beta\|\psi\|_{\Gamma}^{2}+\|\eta\|_{\mathcal{M}_{-1}}^{2}+2(F(u), 1)+2(G(\psi), 1)_{\Gamma} .
$$

It is not difficult to check that the stationary solutions are of the form $\left(u_{*}, \psi_{*}, 0\right)$ where $\left(\left(u_{*}, \psi_{*}\right)\right.$ (formally) solves the boundary value problem

$$
\begin{aligned}
& -\Delta\left(-\Delta u_{*}+f\left(u_{*}\right)\right)=0, \quad \text { in } \Omega \\
& -\Delta_{\Gamma} \psi_{*}+\partial_{\mathbf{n}} u_{*}+\beta \psi_{*}+g\left(\psi_{*}\right)=0, \quad \text { on } \Gamma, \\
& \partial_{\mathbf{n}}\left(-\Delta u_{*}+f\left(u_{*}\right)\right)=0 \quad \text { on } \Gamma .
\end{aligned}
$$

On the other hand, it is known that such a problem admits at least one solution $\left(u_{*}, \psi_{*}\right) \in$ $\mathbb{V}_{3}$ (see, e.g., [51]). Then, denoting by $\mathcal{E}_{M}$ the set of equilibria subject to the constraint $\left|\left\langle u_{*}\right\rangle\right| \leq M$, we have that $\mathcal{E}_{M} \subset \mathcal{A}_{M}$. Moreover, a well-known result (see, e.g., [52]) gives the following characterization of $\mathcal{A}_{M}$

Proposition 4.5 Let the assumptions of Theorem 4.1 be satisfied. Then, the global attractor $\mathcal{A}_{M}$ coincides with the unstable manifold of $\mathcal{E}_{M}$, that is, $\mathcal{A}_{M}$ consists of all points $\Theta_{0}=$ $\left(u_{0}, \psi_{0}, \eta_{0}\right) \in \mathbb{H} \cap \mathcal{H}_{1,0}^{M}$ such that $\Theta(t)=\left(u(t), \psi(t), \eta^{t}\right)$ is a complete bounded trajectory of $S(t)$ originating from $\Theta_{0}$ and

$$
\lim _{t \rightarrow-\infty} \sup _{\Theta_{*} \in \mathcal{E}}\left\|\Theta(t)-\Theta_{*}\right\|_{\mathcal{H}_{1,0}}=0 .
$$

Remark 4.6 The set $\mathcal{E}_{M}$ can be a continuum. Therefore, a given trajectory does not necessarily converge to a single equilibrium. However, if $f$ and $g$ are real analytic, it should be possible to use the Eojasiewicz-Simon technique to show that each trajectory does converge to a unique stationary state (see, e.g., [4, 25, 31, 51] and references therein).

\section{$5 \quad$ Exponential attractors}

Here we establish the existence of exponential attractors in the viscous case. More precisely, we shall prove the following

Theorem 5.1 Let the assumptions of Theorem 4.1 be satisfied. Then, $\left(\mathcal{H}_{1,0}^{M}, S(t)\right)$ possesses an exponential attractor $\mathcal{M}$ which is bounded in $\mathbb{H}$, namely,

(I) $\mathcal{M}$ is compact in $\mathcal{H}_{1,0}$ and positively invariant with respect to $S(t)$, i.e.,

$$
S(t)(\mathcal{M}) \subset \mathcal{M}, \quad \forall t \geq \tau .
$$

(II) The fractal dimension of $\mathcal{M}$ with respect to the $\mathcal{H}_{1,0}$-metric is finite.

$(I I I)$ There exist a positive nondecreasing monotone function $Q$ and a constant $\rho>0$ such that

$$
\operatorname{dist}_{\mathcal{H}_{1,0}}(S(t) B, \mathcal{M}) \leq Q\left(\|B\|_{\mathcal{H}_{1,0}^{M}}\right) e^{-\rho t}, \quad \forall t \geq \tau
$$

where $B$ is any bounded set of initial data in $\mathcal{H}_{1,0}^{M}$. Here dist H $_{1,0}$ denotes the non-symmetric Hausdorff distance in $\mathcal{H}_{1,0}$ and $\|B\|_{\mathcal{H}_{1,0}^{M}}$ stands for the size of $B$ in $\mathcal{H}_{1,0}^{M}$. 
An immediate consequence of Theorem 5.1 is

Corollary 5.2 Let the assumptions of Theorem 4.1 be satisfied. Then, $\mathcal{A}_{M}$ has finite fractal dimension.

We delay the proof of Theorem 5.1 until the end of the section. We first begin with deducing higher-order dissipative estimates.

Lemma 5.3 Let $\Theta_{0}=\left(u_{0}, \psi_{0}, \eta_{0}\right) \in \mathbb{H} \cap \mathcal{H}_{1,0}^{M}$ such that $\left\|\Theta_{0}\right\|_{\mathcal{H}_{1,0}} \leq R$. Then, the following estimate holds:

$$
\left\|S(t) \Theta_{0}\right\|_{\mathbb{H}} \leq Q\left(\left\|\Theta_{0}\right\|_{\mathbb{H}}\right) e^{-\rho_{2}(t-\tau)}+Q(R), \quad \forall t \geq \tau,
$$

for some positive monotone nondecreasing function $Q$ and some positive constant $\rho_{2}$, both independent of time.

Proof. In this proof, $c$ will denote a generic positive constant which is independent of time and $R$ and it may vary even within the same line. We can proceed formally since the argument can be made rigorous by using the approximation scheme developed in the proof of Theorem 3.4 .

Let us set $\widetilde{u}=\partial_{t} u, \widetilde{\psi}=\partial_{t} \psi, \widetilde{\mu}=\partial_{t} \mu, \widetilde{\eta}^{t}=\partial_{t} \eta^{t}$ and recall that $(\widetilde{u}, \widetilde{\psi}, \widetilde{\eta})$ solves problem (3.51)-(3.54). Then identity (3.55) can be rewritten in the following form

$$
\begin{aligned}
& \frac{1}{2} \frac{d}{d t} \mathcal{Y}_{1}(t)-\int_{0}^{\infty} \nu^{\prime}(s)\left\|\widetilde{\eta}^{t}(s)\right\|_{H^{-1}}^{2} d s+\alpha\left\|\partial_{t} \widetilde{u}(t)\right\|^{2}+\left\|\partial_{t} \widetilde{\psi}(t)\right\|_{\Gamma}^{2} \\
& =\frac{1}{2}\left(f^{\prime \prime}(u(t)) \widetilde{u}(t),(\widetilde{u}(t))^{2}\right)+\frac{1}{2}\left(g^{\prime \prime}(u(t)) \widetilde{\psi}(t),(\widetilde{\psi}(t))^{2}\right)_{\Gamma},
\end{aligned}
$$

where

$$
\begin{aligned}
\mathcal{Y}_{1}(t) & =\|\nabla \widetilde{u}(t)\|^{2}+\left\|\nabla_{\Gamma} \widetilde{\psi}(t)\right\|_{\Gamma}^{2}+\beta\|\widetilde{\psi}(t)\|_{\Gamma}^{2}+\left\|\widetilde{\eta}^{t}\right\|_{\mathcal{M}_{-1}}^{2} \\
& +\left(f^{\prime}(u(t)) \widetilde{u}(t), \widetilde{u}(t)\right)+\left(g^{\prime}(u(t)) \widetilde{\psi}(t), \widetilde{\psi}(t)\right)_{\Gamma} .
\end{aligned}
$$

Next, we take $v=\partial_{t} \mu$ in $(3.7), \zeta=A_{N}^{-1} \eta^{t}$ in (3.52) and $\mathbf{v}=(\widetilde{u}(t), \widetilde{\psi})$ in (3.53). Thus, adding together the resulting relationships (note that $\left\langle\widetilde{\eta}^{t}\right\rangle=\langle\widetilde{u}(t)\rangle=0$ ), we find

$$
\begin{aligned}
& \frac{1}{2} \frac{d}{d t}\left[\alpha\|\widetilde{u}(t)\|^{2}+\|\widetilde{\psi}(t)\|_{\Gamma}^{2}+2\left(A_{N}^{-1 / 2} \widetilde{\eta}^{t}, A_{N}^{-1 / 2} \eta^{t}\right)_{\mathcal{M}_{0}}\right]+\|\nabla \widetilde{u}(t)\|^{2}+\left\|\nabla_{\Gamma} \widetilde{\psi}(t)\right\|_{\Gamma}^{2} \\
& +\beta\|\widetilde{\psi}(t)\|_{\Gamma}^{2}+\left(f^{\prime}(u(t)),(\widetilde{u}(t))^{2}\right)+\left(g^{\prime}(\psi(t)),(\widetilde{\psi}(t))^{2}\right)_{\Gamma} \\
& =\left\|\widetilde{\eta}^{t}\right\|_{\mathcal{M}_{-1}}^{2}+\int_{0}^{\infty} \nu^{\prime}(s)\left(A_{N}^{-1 / 2} \eta^{t}(s), A_{N}^{-1 / 2} \widetilde{\eta}^{t}(s)\right) d s .
\end{aligned}
$$


Let us now choose a sufficiently large constant $c_{*}>0$ such that $c_{*}>\max \left\{\left(l_{1}+1\right) / \alpha, l_{2}+1\right\}$, where $l_{1}, l_{2}$ are as in (4.20), and then multiply (5.4) by $c_{*}$ and add to (5.3). This gives

$$
\begin{aligned}
& \frac{1}{2} \frac{d}{d t} \mathcal{Y}_{2}(t)-\int_{0}^{\infty} \nu^{\prime}(s)\left\|\widetilde{\eta}^{t}(s)\right\|_{H^{-1}}^{2} d s+\alpha\left\|\partial_{t} \widetilde{u}(t)\right\|^{2}+\left\|\partial_{t} \widetilde{\psi}(t)\right\|_{\Gamma}^{2} \\
& +c_{*}\left(\|\nabla \widetilde{u}(t)\|^{2}+\left\|\nabla_{\Gamma} \widetilde{\psi}(t)\right\|_{\Gamma}^{2}+\beta\|\widetilde{\psi}(t)\|_{\Gamma}^{2}\right) \\
& +c_{*}\left[\left(f^{\prime}(u(t)),(\widetilde{u}(t))^{2}\right)+\left(g^{\prime}(\psi(t)),(\widetilde{\psi}(t))^{2}\right)_{\Gamma}\right] \\
& =\Lambda_{2}(t),
\end{aligned}
$$

where

$$
\mathcal{Y}_{2}(t)=\mathcal{Y}_{1}(t)+c_{*}\left[\alpha\|\widetilde{u}(t)\|^{2}+\|\widetilde{\psi}(t)\|_{\Gamma}^{2}+2\left(A_{N}^{-1 / 2} \widetilde{\eta}^{t}, A_{N}^{-1 / 2} \eta^{t}\right)_{\mathcal{M}_{0}}\right]
$$

and

$$
\begin{aligned}
\Lambda_{2}(t) & =\frac{1}{2}\left(f^{\prime \prime}(u(t)) \widetilde{u}(t),(\widetilde{u}(t))^{2}\right)+\frac{1}{2}\left(g^{\prime \prime}(u(t)) \widetilde{\psi}(t),(\widetilde{\psi}(t))^{2}\right)_{\Gamma} \\
& +c_{*}\left\|\widetilde{\eta}^{t}\right\|_{\mathcal{M}_{-1}}^{2}+c_{*} \int_{0}^{\infty} \nu^{\prime}(s)\left(A_{N}^{-1 / 2} \eta^{t}(s), A_{N}^{-1 / 2} \widetilde{\eta}^{t}(s)\right) d s .
\end{aligned}
$$

Thanks to (4.17) and (4.20), the growth assumptions (N2') and Young's inequality, we easily see that $\mathcal{Y}_{2}(t)$ fulfills the inequalities

$$
c\left\|\left(\widetilde{u}(t), \widetilde{\psi}(t), \widetilde{\eta}^{t}\right)\right\|_{\mathcal{H}_{1,0}}^{2}-C_{0}^{\prime}(R) \leq \mathcal{Y}_{2}(t) \leq c\left\|\left(\widetilde{u}(t), \widetilde{\psi}(t), \widetilde{\eta}^{t}\right)\right\|_{\mathcal{H}_{1,0}}^{2}+C_{0}^{\prime}(R),
$$

for all $t \geq \tau$, where $C_{0}^{\prime}$ depends on $C_{0}$ (see (4.17)). Let us now estimate the first two nonlinear terms in $\Lambda_{2}(t)$. By the standard Hölder and Sobolev inequalities, we have

$$
\begin{aligned}
& \frac{1}{2}\left(f^{\prime \prime}(u(t)) \widetilde{u}(t),(\widetilde{u}(t))^{2}\right)+\frac{1}{2}\left(g^{\prime \prime}(u(t)) \widetilde{\psi}(t),(\widetilde{\psi}(t))^{2}\right)_{\Gamma} \\
& \leq c\left(\|u(t)\|_{H^{1}}\left\|\partial_{t} u(t)\right\|\|\widetilde{u}(t)\|_{H^{1}}^{2}+\|\psi(t)\|_{H^{1}(\Gamma)}\left\|\partial_{t} \psi(t)\right\|_{\Gamma}\|\widetilde{\psi}(t)\|_{H^{1}(\Gamma)}^{2}\right),
\end{aligned}
$$

for all $t \geq \tau$. Besides, on account of (K5) and (4.17), for all $t \geq \tau$, we also have

$$
\int_{0}^{\infty} \nu^{\prime}(s)\left(A_{N}^{-1 / 2} \eta^{t}(s), A_{N}^{-1 / 2} \widetilde{\eta}^{t}(s)\right) d s \leq c\left\|\widetilde{\eta}^{t}\right\|_{\mathcal{M}_{-1}}^{2}+C_{0}(R) .
$$

Hence, combining (5.7) and (5.8) with (5.4), we end up with the following differential inequality:

$$
\begin{aligned}
& \frac{1}{2} \frac{d}{d t} \mathcal{Y}_{2}(t)+c_{*} \mathcal{Y}_{2}(t) \\
\leq & c\left(1+\|u(t)\|_{H^{1}}\left\|\partial_{t} u(t)\right\|+\|\psi(t)\|_{H^{1}(\Gamma)}\left\|\partial_{t} \psi(t)\right\|_{\Gamma}\right) \mathcal{Y}_{2}(t)+C_{0}(R),
\end{aligned}
$$


for all $t \geq \tau$. Due to the estimates (4.1) and (4.17), we easily deduce

$$
\begin{aligned}
& \int_{\tau}^{t}\left(1+\|u(y)\|_{H^{1}}\left\|\partial_{t} u(y)\right\|+\|\psi(y)\|_{H^{1}(\Gamma)}\left\|\partial_{t} \psi(y)\right\|_{\Gamma}\right) d y \\
& \leq c(t-\tau)+C_{0}^{\prime \prime}(R), \quad \forall t \geq \tau,
\end{aligned}
$$

where $C_{0}^{\prime \prime}>0$ depends on $C_{0}$. Using once more Gronwall's inequality (see, e.g., [7, Lemma $3.5]$ ), on account of (5.6) and (5.10), from (5.9) we get

$$
\left\|\left(\widetilde{u}(t), \widetilde{\psi}(t), \widetilde{\eta}^{t}\right)\right\|_{\mathcal{H}_{1,0}}^{2} \leq Q_{1}\left(\left\|\left(\widetilde{u}(\tau), \widetilde{\psi}(\tau), \widetilde{\eta}^{\tau}\right)\right\|_{\mathcal{H}_{1,0}}^{2}\right) e^{-c(t-\tau)}+Q_{1}(R),
$$

for all $t \geq \tau$ and some positive monotone nondecreasing function $Q_{1}$. Arguing now, as in end of the proof of Theorem 4.4, it is not difficult to realize that

$$
\left\|\left(u(t), \psi(t), \eta^{t}\right)\right\|_{\mathcal{H}_{3,2}}^{2}+\|\mu(t)\|_{H^{1}}^{2} \leq Q_{2}\left(\left\|\left(\widetilde{u}(\tau), \widetilde{\psi}(\tau), \widetilde{\eta}^{\tau}\right)\right\|_{\mathcal{H}_{1,0}}^{2}\right) e^{-c(t-\tau)}+Q_{2}(R)
$$

for all $t \geq \tau$ and some positive monotone nondecreasing function $Q_{2}$. On the other hand, we have (cf. (3.49)-(3.50))

$$
\left\|\left(\widetilde{u}(\tau), \widetilde{\psi}(\tau), \widetilde{\eta}^{\tau}\right)\right\|_{\mathcal{H}_{1,0}} \leq Q_{3}\left(\left\|\left(u(\tau), \psi(\tau), \eta^{\tau}\right)\right\|_{\mathcal{H}_{3,2}}\right)
$$

for some positive monotone nondecreasing function $Q_{3}$. On account of (5.12)-(5.13), we can also control the remaining terms of (4.18) using [8, Lemma 3.4]. The proof is complete.

The next lemma is concerned with the time regularity of the semigroup $S(t)$.

Lemma 5.4 Let the assumptions of Theorem 4.1 be satisfied. For any $R>0$, there exist a positive constant $C=C(R)$ and a time $t^{*}=t^{*}(R)>\tau$ such that

$$
\left\|S(t) \Theta_{0}-S(\tilde{t}) \Theta_{0}\right\|_{\mathcal{H}_{1,0}} \leq C|t-\tilde{t}|^{1 / 2}
$$

for all $t, \tilde{t} \in\left[t^{*}, \infty\right)$ and any $\Theta_{0} \in B_{\mathcal{H}_{3,2}}(R) \subset \mathcal{H}_{1,0}$.

Proof. Estimate (5.2) (cf. also (5.11)) entails

$$
\left\|\left(\partial_{t} u(t), \partial_{t} \psi(t), \partial_{t} \eta^{t}\right)\right\|_{\mathbb{V}_{1} \times \mathcal{M}_{-1}} \leq C,
$$

for all $t \geq \tau$. Observing that

$$
S(t) \Theta_{0}-S(\tilde{t}) \Theta_{0}=\int_{t}^{\tilde{t}} \partial_{y}\left(S(y) \Theta_{0}\right) d y,
$$

thanks to (5.15), we deduce (5.14).

The second lemma is devoted to show that $S(t)$ has the so-called smoothing property (see $[40,3.2])$. 
Lemma 5.5 Let the assumptions of Theorem 4.1 be satisfied. Fix $R>0$ and let $\left(u_{i}, \psi_{i}, \eta_{i}\right)$ be two trajectories corresponding to two initial data $\Theta_{0 i}=\left(u_{0 i}, \psi_{0 i}, \eta_{0 i}\right) \in B_{\mathcal{H}_{3,2}}^{M}(R), i=1,2$, respectively. Then the following decomposition holds

$$
S(t) \Theta_{01}-S(t) \Theta_{02}=D(t)\left(\Theta_{01}, \Theta_{02}\right)+N(t)\left(\Theta_{01}, \Theta_{02}\right)
$$

wher, for all $t \geq \tau$, we have

$$
\begin{gathered}
\left\|D(t)\left(\Theta_{01}, \Theta_{02}\right)\right\|_{\mathcal{H}_{1,0}} \leq \kappa e^{-\kappa(t-\tau)}\left\|\Theta_{01}-\Theta_{02}\right\|_{\mathcal{H}_{1,0}} \\
\left\|N(t)\left(\Theta_{01}, \Theta_{02}\right)\right\|_{\mathbb{H}} \leq K e^{K(t-\tau)}\left\|\Theta_{01}-\Theta_{02}\right\|_{\mathcal{H}_{1,0}}
\end{gathered}
$$

for some positive constants $\kappa$ and $K$.

Proof. Also in this proof we will argue formally on account of the approximation scheme exploited in Section 3. We begin by observing that, for each $\Theta_{0} \in B_{\mathcal{H}_{3,2}}^{M}(R)$, from (N2') and (5.2), thanks to the embeddings $H^{2}(\Omega) \hookrightarrow L^{\infty}(\Omega)$ and $H^{2}(\Gamma) \hookrightarrow L^{\infty}(\Gamma)$, we have that

$$
\left.\sum_{i=0}^{2}\left(\left\|f^{(i)}(u(t))\right\|_{L^{\infty}}+\| g^{(i)}(\psi(t))\right) \|_{L^{\infty}(\Gamma)}\right) \leq C_{R},
$$

for some positive constant $C_{R}$ and all $t \geq \tau$. We now write a given trajectory as follows (recall that $\left.S(t) \Theta_{0}=\left(u(t), \psi(t), \eta^{t}\right)\right)$

$$
S(t) \Theta_{0}=\widetilde{D}(t) \Theta_{0}+\widetilde{N}(t) \Theta_{0},
$$

where

$$
\widetilde{D}(t) \Theta_{0}=\left(w_{d}(t), \zeta_{d}(t), \xi_{d}^{t}\right), \widetilde{N}(t) \Theta_{0}=\left(w_{c}(t), \zeta_{c}(t), \xi_{c}^{t}\right)
$$

are solutions to the following problems, respectively,

$$
\begin{aligned}
& \partial_{t} w_{d}+\int_{0}^{\infty} \nu(s) \xi_{d}(s) d s=0, \\
& \Upsilon\left(\mathbf{w}_{d}, \mathbf{v}\right)_{\mathbb{V}_{1}^{*}, \mathbb{V}_{1}}+\left(\alpha \partial_{t} w_{d}, v\right)+\left(\partial_{t} \zeta_{d}, \varphi\right)_{\Gamma}=\left(\mu_{d}, v\right)_{H^{-1}, H^{1}}, \quad \forall \mathbf{v}=(v, \varphi) \in \mathbb{V}_{1}, \\
& \partial_{t} \xi_{d}-\mathbb{T} \xi_{d}=A_{N} \mu_{d}, \\
& w_{d}(\tau)=u_{0}-\left\langle u_{0}\right\rangle, \quad \zeta_{d}(\tau)=\psi_{0}, \quad \xi_{d}^{\tau}=\eta_{0},
\end{aligned}
$$

and

$$
\begin{aligned}
& \partial_{t} w_{c}+\int_{0}^{\infty} \nu(s) \xi_{c}(s) d s=0, \\
& \Upsilon\left(\mathbf{w}_{c}, \mathbf{v}\right)_{\mathbb{V}_{1}^{*}, \mathbb{V}_{1}}+(f(u), v)+(g(\psi), \varphi)_{\Gamma}+\left(\alpha \partial_{t} w_{c}, v\right)+\left(\partial_{t} \zeta_{c}, \varphi\right)_{\Gamma} \\
& =\left(\mu_{c}, v\right)_{H^{-1}, H^{1}}, \quad \forall \mathbf{v}=(v, \varphi) \in \mathbb{V}_{1}, \\
& \partial_{t} \xi_{c}-\mathbb{T} \xi_{c}=A_{N} \mu_{c}, \\
& w_{c}(\tau)=\left\langle u_{0}\right\rangle, \quad \zeta_{c}(\tau)=0, \quad \xi_{c}^{\tau}=0 .
\end{aligned}
$$


Then, for each $\Theta_{0 i}=\left(u_{0 i}, \psi_{0 i}, \eta_{0 i}\right) \in B_{\mathcal{H}_{3,2}}^{M}(R), i=1,2$, we define the operators in (5.17), as follows:

$$
\begin{aligned}
& D(t)\left(\Theta_{01}, \Theta_{02}\right)=\widetilde{D}(t) \Theta_{01}-\widetilde{D}(t) \Theta_{02} \\
& N(t)\left(\Theta_{01}, \Theta_{02}\right)=\widetilde{N}(t) \Theta_{01}-\widetilde{N}(t) \Theta_{02}
\end{aligned}
$$

The first assertion of (5.18) is now immediate, since it is easy to realize that $\widetilde{D}(t)$ is an exponentially stable linear strongly continuous semigroup on the Hilbert space $\mathcal{H}_{1,0}^{0}$. Thus, it remains to show that (5.19) holds.

We proceed as in the proof of Theorem 4.4. We begin by first noticing that

$$
(\bar{w}, \bar{\zeta}, \bar{\xi})=\left(w_{1 c}-w_{2 c}, \zeta_{1 c}-\zeta_{2 c}, \xi_{1 c}-\xi_{2 c}\right)=N\left(\Theta_{01}, \Theta_{02}\right)
$$

satisfies the following system:

$$
\begin{aligned}
& \partial_{t} \bar{w}+\int_{0}^{\infty} \nu(s) \bar{\xi}^{t}(s) d s=0, \\
& \Upsilon((\bar{w}, \bar{\zeta}), \mathbf{v})_{\mathbb{V}_{1}^{*}, \mathbb{V}_{1}}+\left(f\left(u_{1}\right)-f\left(u_{2}\right), \bar{v}\right)+\left(g\left(\psi_{1}\right)-g\left(\psi_{2}\right), \varphi\right)_{\Gamma}+ \\
& +\left(\alpha \partial_{t} \bar{w}, v\right)+\left(\partial_{t} \bar{\zeta}, \varphi\right)_{\Gamma}=(\bar{\mu}, v)_{H^{-1}, H^{1}}, \quad \forall \mathbf{v}=(v, \varphi) \in \mathbb{V}_{1}, \\
& \partial_{t} \bar{\xi}^{t}-\mathbb{T}^{t} \bar{\xi}^{t}=A_{N} \bar{\mu}, \\
& \bar{w}(\tau)=\left\langle u_{01}-u_{02}\right\rangle, \quad \bar{\zeta}(\tau)=0, \quad \bar{\xi}^{\tau}=0,
\end{aligned}
$$

Arguing as in the proof of Lemma 5.3 and exploiting the (3.62) together with (5.20), it is not difficult to deduce that

$$
\frac{d}{d t}\left\|N(t)\left(\Theta_{01}, \Theta_{02}\right)\right\|_{\mathcal{H}_{3,2}}^{2} \leq C_{R}^{\prime}\left\|N(t)\left(\Theta_{01}, \Theta_{02}\right)\right\|_{\mathcal{H}_{3,2}}^{2}+C_{R}^{\prime \prime}\left\|\Theta_{01}-\Theta_{02}\right\|_{\mathcal{H}_{1,0}}^{2}
$$

for all $t \geq \tau$, for some positive constants $C_{R}^{\prime}$ and $C_{R}^{\prime \prime}$. Recalling that

$$
N(0)\left(\Theta_{01}, \Theta_{02}\right)=\left(\left\langle u_{01}-u_{02}\right\rangle, 0,0\right)
$$

and the standard Gronwall inequality gives

$$
\left\|N(t)\left(\Theta_{01}, \Theta_{02}\right)\right\|_{\mathcal{H}_{3,2}}^{2} \leq K e^{K(t-\tau)}\left\|\Theta_{01}-\Theta_{02}\right\|_{\mathcal{H}_{1,0}}^{2}
$$

for some positive constant $K$. On the other hand, using once more [8, Lemma 3.4], we obtain

$$
\left\|\mathbb{T} \bar{\xi}^{t}\right\|_{\mathcal{M}_{-1}}^{2}+\sup _{x \geq 1} x \int_{(x, \infty)} \nu(s)\left\|\bar{\xi}^{t}(s)\right\|_{H^{-1}}^{2} d s \leq K e^{K(t-\tau)}\left\|\Theta_{01}-\Theta_{02}\right\|_{\mathcal{H}_{1,0}}^{2},
$$

for all $t \geq \tau$. Thus, (5.34)-(5.35) yields (5.19).

We can now conclude the section with the proof of the main theorem.

Proof of Theorem 5.1. Thanks to Theorem 4.4 and Lemma 5.3 we can find a bounded set $\mathbb{B} \subset \mathbb{H}$ which is positively invariant for $S(t)$ and attracts any bounded set of $\mathcal{H}_{1,0}^{M}$ exponentially fast. Moreover, the map $\left(t, \Theta_{0}\right) \longmapsto S(t) \Theta_{0}$ is Hölder continuous on $[\tau, T] \times \mathbb{B}$, provided that $\mathbb{B}$ is endowed with the $\mathcal{H}_{1,0}$-topology. Also, Lemma 5.5 shows that $S(t)$ enjoys the smoothing property. Thus, on account of a well-known result (see [40, Thm. 3.7, Rem.3.8] and its references), the existence of an exponential attractor follows. 


\section{Trajectory attractors}

In this section we would like consider the case $\alpha=0$. We remind that in this case we only have the existence of a variational solution given by Theorem 3.3. We already mentioned that uniqueness is a difficult issue which is still open in dimension three, even for exponential kernels (see [27, 28]). Thus here we will employ the trajectory approach (see [3], cf. also [40]) to establish the existence of the trajectory attractor.

We begin with the following consequence of Theorem 3.3 and Lemma 4.2, based on the construction of a solution through the Galerkin scheme introduced in Section 3.

Corollary 6.1 Let (K1)-(K5) and (N0), (N1), (N2) hold. Fix a number $N>0$ and let us define the trajectory space $\mathcal{K}_{N}$ as the union of all weak solutions $(u, \psi, \eta)$ of problem $\boldsymbol{P}$ that satisfy the inequality

$$
\left\|\left(u(t), \psi(t), \eta^{t}\right)\right\|_{\mathcal{H}_{1,0}}^{2} \leq Q(N) e^{-\rho(t-\tau)}+C_{M}, \quad \forall t \geq \tau,
$$

where $\tau \in \mathbb{R}$ and $Q$ is a positive monotone increasing function. Then, for any $\left(u_{0}, \psi_{0}, \eta_{0}\right) \in$ $\mathcal{H}_{1,0}^{M}$ such that $\left\|\left(u_{0}, \psi_{0}, \eta_{0}\right)\right\|_{\mathcal{H}_{1,0}} \leq N$, there exists at least one trajectory $\left(u(y), \psi(y), \eta^{y}\right) \in$ $\mathcal{K}_{N}$ such that $u(\tau)=u_{0}, \psi(\tau)=\psi_{0}$ and $\eta^{\tau}=\eta_{0}$.

In order to define the dynamical system we need to introduce first the appropriate functional framework following [3]. To this end, we consider the space

$$
\begin{aligned}
\mathcal{Z}[\tau, T]:=\{ & (u, \psi, \eta) \in L^{\infty}\left([\tau, T] ; \mathcal{H}_{1,0}^{M}\right): A_{\Gamma} u \in L^{\infty}\left([\tau, T] ; \mathbb{V}_{1}^{*}\right), \\
& \left.\partial_{t} u \in L^{\infty}\left([\tau, T] ;\left(H^{1}(\Omega)\right)^{*}\right), \partial_{t} \psi \in L^{2}\left([\tau, T] ; L^{2}(\Gamma)\right)\right\},
\end{aligned}
$$

for any fixed $T>\tau$, endowed with the following norm

$$
\begin{aligned}
\|(u, \psi, \eta)\|_{\mathcal{Z}[\tau, T]}^{2} & =\|(u, \psi)\|_{L^{\infty}\left([\tau, T] ; \mathbb{V}_{1}\right)}^{2}+\|\eta\|_{L^{\infty}([\tau, T] ; \mathcal{M}-1)}^{2}+\left\|A_{\Gamma} u\right\|_{L^{\infty}\left([\tau, T] ; \mathbb{V}_{1}^{*}\right)}^{2} \\
& +\left\|\partial_{t} u\right\|_{L^{\infty}\left([\tau, T] ;\left(H^{1}(\Omega)\right)^{*}\right)}^{2}+\left\|\partial_{t} \psi\right\|_{L^{2}\left([\tau, T] ; L^{2}(\Gamma)\right)}^{2} .
\end{aligned}
$$

It is easy to see that $\mathcal{Z}[\tau, T]$ is Banach space. Moreover, we also introduce the Banach space $\mathcal{Z}_{b}^{+}$defined by

$$
\begin{aligned}
\mathcal{Z}_{b}^{+}:=\left\{(u, \psi, \eta) \in L^{\infty}\left((\tau, \infty) ; \mathcal{H}_{1,0}^{M}\right): A_{\Gamma} u \in L^{\infty}\left((\tau, \infty) ; \mathbb{V}_{1}^{*}\right),\right. \\
\left.\partial_{t} u \in L^{\infty}\left((\tau, \infty) ;\left(H^{1}(\Omega)\right)^{*}\right), \partial_{t} \psi \in L_{b}^{2}\left((\tau, \infty) ; L^{2}(\Gamma)\right)\right\},
\end{aligned}
$$

and the function space $\mathcal{Z}_{l o c}^{+}$defined as follows

$$
\begin{aligned}
& \mathcal{Z}_{l o c}^{+}:=\left\{(u, \psi, \eta) \in L_{l o c}^{\infty}\left((\tau, \infty) ; \mathcal{H}_{1,0}^{M}\right): A_{\Gamma} u \in L_{l o c}^{\infty}\left((\tau, \infty) ; \mathbb{V}_{1}^{*}\right),\right. \\
&\left.\partial_{t} u \in L_{l o c}^{\infty}\left((\tau, \infty) ;\left(H^{1}(\Omega)\right)^{*}\right), \partial_{t} \psi \in L_{l o c}^{2}\left((\tau, \infty) ; L^{2}(\Gamma)\right)\right\} .
\end{aligned}
$$

The space $\mathcal{Z}_{l o c}^{+}$is the inductive limit of the topological spaces $\{\Xi[\tau, T]\}_{T>\tau}$ defined here below. 
Definition 6.2 We denote by $\Xi[\tau, T]$ the space $\mathcal{Z}[\tau, T]$ endowed with the convergence topology: a sequence $\left(u_{n}, \psi_{n}, \eta_{n}\right) \in \mathcal{Z}[\tau, T]$ converge to $(u, \psi, \eta)$ if and only if $\left(u_{n}, \psi_{n}, \eta_{n}\right) \rightarrow$ $(u, \psi, \eta)$ weakly star in $L^{\infty}\left([\tau, T] ; \mathcal{H}_{1,0}^{M}\right), A_{\Gamma} u_{n} \rightarrow A_{\Gamma} u$ weakly star in $L^{\infty}\left((\tau, \infty) ; \mathbb{V}_{1}^{*}\right)$, $\partial_{t} u_{n} \rightarrow \partial_{t} u$ weakly star in $L^{2}\left([\tau, T] ;\left(H^{1}(\Omega)\right)^{*}\right)$ and $\partial_{t} \psi_{n} \rightarrow \partial_{t} \psi$ weakly in $L^{2}\left([\tau, T] ; L^{2}(\Gamma)\right)$.

We recall that $\mathcal{Z}_{\text {loc }}^{+}$is a Hausdorff and Fréchet-Urysohn space with a countable topology base (see [3, Chap. XII]). On account of Corollary 6.1, for any $N>0$, we deduce

$$
\mathcal{K}_{N} \subset \mathcal{Z}_{b}^{+} \subset \mathcal{Z}_{\text {loc }}^{+} .
$$

Note that the space $\mathcal{K}_{N}$ is a topological space when endowed with the topology of $\mathcal{Z}_{\text {loc }}^{+}$and $\mathcal{K}_{N}$ is closed in $\mathcal{Z}_{\text {loc }}^{+}$(see the proof of Lemma 4.2). This allows us to define the trajectory dynamical system associated with problem $\mathbf{P}$ in the sense of Definition 3.1. More precisely, we introduce the translation semigroup $\{\mathcal{T}(t), t \geq \tau\}$ acting on $\mathcal{Z}_{\text {loc }}^{+}$as follows

$$
\mathcal{T}(t)\left(u(\cdot), \psi(\cdot), \eta^{(\cdot)}\right)=\left(u(t+\cdot), \psi(t+\cdot), \eta^{(t+\cdot)}\right), \quad t \geq \tau .
$$

Arguing as in [3, Chapter XII], we can prove the following.

Theorem 6.3 Let the assumptions of Corollary 6.1 hold. Then $\{\mathcal{T}(t): t \geq \tau\}$ is continuous in the topological space $\mathcal{Z}_{\text {loc }}^{+}$and

$$
\mathcal{T}(t)\left(\mathcal{K}_{N}\right) \subseteq \mathcal{K}_{N}, \quad \forall t \geq \tau .
$$

We recall the definition of trajectory attractor in our case.

Definition 6.4 The trajectory attractor of the semigroup $\{\mathcal{T}(t), t \geq \tau\}$ on $\mathcal{K}_{N}$ is a set $\mathcal{X}_{\text {tr }} \subset \mathcal{K}_{N}$ such that:

(i) $\mathcal{X}_{\text {tr }}$ is compact in $\mathcal{Z}_{\text {loc }}^{+}$and bounded in $\mathcal{Z}_{b}^{+}$;

(ii) $\mathcal{X}_{t r}$ is strictly invariant, that is,

$$
\mathcal{T}(t) \mathcal{X}_{t r}=\mathcal{X}_{t r}, \quad \forall t \geq \tau
$$

(iii) $\mathcal{X}_{t r}$ is a uniformly attracting set for the semigroup $\{\mathcal{T}(t), t \geq \tau\}$, that is, for every neighborhood $\mathcal{O}=\mathcal{O}\left(\mathcal{X}_{t r}\right)$ in the topology of $\mathcal{K}_{N}$, there exists $t^{+} \geq \tau$ such that

$$
\mathcal{T}(t)\left(\mathcal{X}_{t r}\right) \subseteq \mathcal{O}, \quad \forall t \geq t^{+} .
$$

Let $\Pi_{+}$denote the restriction operator on $(\tau, \infty)$ and consider the set

$$
\Psi=\left\{(u, \psi, \eta) \in \mathcal{Z}_{b}^{+}:\left\|\left(u(t), \psi(t), \eta^{t}\right)\right\|_{\mathcal{H}_{1,0}}^{2} \leq 2 C_{M}, \quad \forall t \geq \tau\right\} .
$$

Note that $\Psi$ is bounded in $\mathcal{Z}_{b}^{+}$, compact in $\mathcal{Z}_{\text {loc }}^{+}$and, owing to (6.1), it is also an attracting (and even absorbing) set for the space $\mathcal{K}_{N}$, for any $N>0$. On account of [3, Chapter 
XII, Definition 2.5], we can associate with problem $\mathbf{P}$, the kernel $\mathcal{K}$. This set consists of all complete trajectories $(u, \psi, \eta)$ that are bounded in the space

$$
\begin{aligned}
\mathcal{Z}_{b}:= & \left\{(u, \psi, \eta) \in L^{\infty}\left(\mathbb{R} ; \mathcal{H}_{1,0}^{M}\right):\right. \\
& \left.A_{\Gamma} u \in L^{\infty}\left(\mathbb{R} ; \mathbb{V}_{1}^{*}\right), \partial_{t} u \in L^{\infty}\left(\mathbb{R} ;\left(H^{1}(\Omega)\right)^{*}\right), \partial_{t} \psi \in L_{b}^{2}\left(\mathbb{R} ; L^{2}(\Gamma)\right)\right\}
\end{aligned}
$$

and satisfy the inequality

$$
\left\|\left(u(t), \psi(t), \eta^{t}\right)\right\|_{\mathcal{H}_{1,0}}^{2} \leq C_{M}, \quad \forall t \in \mathbb{R} .
$$

Indeed, observe that a complete trajectory satisfies

$$
\Pi_{+}\left(u(h+y-\tau), \psi(h+y-\tau), \eta^{h+y-\tau}\right) \in \mathcal{K}_{N}
$$

for all $h \in \mathbb{R}$. Therefore by $(6.1)$,

$$
\left\|\left(u(h+y-\tau), \psi(h+y-\tau), \eta^{h+y-\tau}\right)\right\|_{\mathcal{H}_{1,0}}^{2} \leq Q(N) e^{-\rho(y-\tau)}+C_{M}, \quad \forall y \geq \tau
$$

Taking $h=t-y+\tau$ and letting $y \rightarrow \infty$, we obtain (6.9).

Thus a consequence of [3, Chapter XII, Theorems 2.1, 2.2] is

Theorem 6.5 Let the assumptions of Corollary 6.1 hold. Then the translation semigroup $\{\mathcal{T}(t), t \geq \tau\}$ acting on $\mathcal{K}_{N}$ possesses the trajectory attractor $\mathcal{X}_{t r}$ which satisfies properties (i)-(iii) of Definition 6.4, is bounded in $\mathcal{Z}_{b}^{+}$and compact in $\mathcal{Z}_{\text {loc }}^{+}$. Moreover, $\mathcal{X}_{t r}$ is independent of $N$ (since $\mathcal{K}$ does) and

$$
\mathcal{X}_{t r}=\Pi_{+} \mathcal{K} .
$$

The set $\mathcal{K}$ is bounded in $\mathcal{Z}_{b}$ and compact in $\mathcal{Z}_{\text {loc }}$.

\section{References}

[1] J.W. Cahn, J.E. Hilliard, Free energy of a nonuniform system. I. Interfacial energy, J. Chem. Phys. 28 (1958), 258-267.

[2] C. Cavaterra, C.G. Gal, M. Grasselli, A. Miranville, Phase-field systems with nonlinear coupling and dynamic boundary conditions, Nonlinear Analysis 72 (2010), 2375-2399.

[3] V.V. Chepyzhov, M.I. Vishik, Attractors for equations of mathematical physics, Amer. Math. Soc., Providence, RI, 2002.

[4] R. Chill, E. Fašangová, J. Prüss, Convergence to steady states of solutions of the CahnHilliard and Caginalp equations with dynamic boundary conditions, Math. Nachr. 13 (2006), 1448-1462. 
[5] M. Conti, M. Coti Zelati, Attractors for the non-viscous Cahn-Hilliard with memory in 2D, Nonlinear Anal. 72 (2010), 1668-1682.

[6] M. Conti, S. Gatti, M. Grasselli, V. Pata, Two-dimensional reaction-diffusion equations with memory, Quart. Appl. Math., to appear.

[7] M. Conti, G. Mola, 3-D viscous Cahn-Hilliard equation with memory, Math. Meth. Appl. Sci. 32 (2009), 1370-1395.

[8] M. Conti, V. Pata, M. Squassina, Singular limit of differential system with memory, Indiana University Mathematics Journal 55 (2006), 169-216.

[9] M. Efendiev, A. Miranville, S. Zelik, Exponential attractors for a singularly perturbed Cahn-Hilliard system, Math. Nachr. 272 (2004), 11-31.

[10] H.P. Fischer, Ph. Maass, W. Dieterich, Novel surface modes of spinodal decomposition, Phys. Rev. Letters 79 (1997), 893-896.

[11] H.P. Fischer, Ph. Maass, W. Dieterich, Diverging time and length scales of spinodal decomposition modes in thin flows, Europhys. Letters 42 (1998), 49-54.

[12] C.G. Gal, Well-posedness and long time behavior of the non-isothermal viscous CahnHilliard model with dynamic boundary conditions, Dyn. Partial Differ. Equ. 5 (2008), 39-67.

[13] C.G. Gal, G.R. Goldstein, J.A. Goldstein, S. Romanelli, M. Warma, Fredholm alternative, semilinear elliptic problems, and Wentzell boundary conditions, submitted.

[14] C.G. Gal, A. Miranville, Uniform global attractors for non-isothermal Cahn-Hilliard equations with dynamic boundary conditions, Nonlinear Anal. Real World Appl. 10 (2009), no. 3, 1738-1766.

[15] P. Galenko, D. Jou, Diffuse-interface model for rapid phase transformations in nonequilibrium systems, Phys. Rev. E 71 (2005), 046125 (13 pages).

[16] P. Galenko, D. Jou, Kinetic contribution to the fast spinodal decomposition controlled by diffusion, Phys. A 388 (2009) 3113-3123.

[17] P. Galenko, V. Lebedev, Analysis of the dispersion relation in spinodal decomposition of a binary system, Philos. Mag. Lett. 87 (2007), 821-827.

[18] P. Galenko, V. Lebedev, Local nonequilibrium effect on spinodal decomposition in a binary system, Int. J. Thermodyn. 11 (2008), 21-28.

[19] P. Galenko, V. Lebedev, Nonequilibrium effects in spinodal decomposition of a binary system, Phys. Lett. A 372 (2008), 985-989. 
[20] S. Gatti, M. Grasselli, A. Miranville, V. Pata, Hyperbolic relaxation of the viscous Cahn-Hilliard equation in 3D, Math. Models Methods Appl. Sci. 15 (2005), 165-198.

[21] S. Gatti, M. Grasselli, A. Miranville, V. Pata, Memory relaxation of first order evolution equations, Nonlinearity 18 (2005), 1859-1883.

[22] S. Gatti, M. Grasselli, A. Miranville, V. Pata, Memory relaxation of the one-dimensional Cahn-Hilliard equation. Dissipative phase transitions, 101-114, Ser. Adv. Math. Appl. Sci., 71, World Sci. Publ., Hackensack, NJ, 2006.

[23] G. Gilardi, On a conserved phase field model with irregular potentials and dynamic boundary conditions, Istit. Lombardo Accad. Sci. Lett. Rend. A 141 (2007), 129-161.

[24] G. Gilardi, A. Miranville, G. Schimperna, On the Cahn-Hilliard equation with irregular potentials and dynamic boundary conditions, Comm. Pure Appl. Anal. 8 (2009), 881912.

[25] M. Grasselli, On the large time behavior of a phase-field system with memory, Asymptotic Analysis 56 (2008), 229-249.

[26] M. Grasselli, V. Pata, F.M. Vegni, Longterm dynamics of a conserved phase-field system with memory, Asymptotic Anal. 33 (2003), 261-320.

[27] M. Grasselli, G. Schimperna, A. Segatti, S. Zelik, On the 3D Cahn-Hilliard equation with inertial term, J. Evol. Equ. 9, 371-404, 2009.

[28] M. Grasselli, G. Schimperna, S. Zelik, On the 2D Cahn-Hilliard equation with inertial term, Comm. Partial Differential Equations 34 (2009), 137-170.

[29] M. Grasselli, G. Schimperna, S. Zelik, Trajectory and smooth attractors for CahnHilliard equations with inertial term, Nonlinearity 23 (2010), 707-737.

[30] M. Grasselli, V. Pata, Uniform attractors of nonautonomous dynamical systems with memory, in "Evolution Equations, Semigroups and Functional Analysis" (A. Lorenzi and B. Ruf, Eds.), Progr. Nonlinear Differential Equations Appl. Birkhäuser, Boston 50 (2002), 155-178.

[31] M. Grasselli, H. Petzeltová, G. Schimperna, Asymptotic behaviour of a non-isothermal viscous Cahn-Hilliard equation with inertial term, J. Differential Equations 239 (2007), $38-60$.

[32] M.E. Gurtin, Generalized Ginzburg-Landau and Cahn-Hilliard equations based on a microforce balance, Phys. D 92 (1996), 178-192.

[33] M. B. Kania, Global attractor for the perturbed viscous Cahn-Hilliard equation, Colloq. Math. 109 (2007), no. 2, 217-229. 
[34] M.B. Kania, Upper semicontinuity of global attractors for the perturbed viscous CahnHilliard equations, Topol. Methods Nonlinear Anal. 32 (2008), no. 2, 327-345.

[35] T. Kato, Perturbation Theory for Linear Operators, corr. printing of the 2nd ed., Springer, Berlin, 1980.

[36] R. Kenzler, F. Eurich, Ph. Maass, B. Rinn, J. Schropp, E. Bohl, W. Dieterich, Phase separation in confined geometries: Solving the Cahn-Hilliard equation with generic boundary conditions, Computer Phys. Comm. 133 (2001), 139-157.

[37] N. Lecoq, H. Zapolsky, P. Galenko, Evolution of the structure factor in a hyperbolic model of spinodal decomposition, Eur. Phys. J. Special Topics 177 (2009), 165-175.

[38] A. Lorenzi, E. Rocca, Weak solutions for the fully hyperbolic phase-field system of conserved type, J. Evol. Equ. 7 (2007), no. 1, 59-78.

[39] A. Miranville, S. Zelik, Exponential attractors for the Cahn-Hilliard equation with dynamic boundary conditions, Math. Models Appl. Sci. 28 (2005), 709-735.

[40] A. Miranville, S. Zelik, Attractors for dissipative partial differential equations in bounded and unbounded domains. Handbook of differential equations: evolutionary equations. Vol. IV, 103-200, Handb. Differ. Equ., Elsevier/North-Holland, Amsterdam, 2008.

[41] A. Miranville, S. Zelik, The Cahn-Hilliard equation with singular potentials and dynamic boundary conditions, Discrete Contin. Dyn. Syst., to appear.

[42] A. Novick-Cohen, On the viscous Cahn-Hilliard equation, in "Material instabilities in continuum mechanics (Edinburgh, 1985-1986)", Oxford Sci. Publ., 329-342, Oxford Univ. Press, New York, 1988.

[43] A. Novick-Cohen, The Cahn-Hilliard equation, Evolutionary equations. Vol. IV, 201228, Handb. Differ. Equ., Elsevier/North-Holland, Amsterdam, 2008.

[44] V. Pata, S. Zelik, A remark on the damped wave equation, Commun. Pure Appl. Anal. 5 (2006), 609-614.

[45] V. Pata, A. Zucchi, Attractors for a damped hyperbolic equation with linear memory, Adv. Math. Sci. Appl. 11 (2001), 505-529.

[46] J. Prüss, R. Racke, S. Zheng, Maximal regularity and asymptotic behavior of solutions for the Cahn-Hilliard equation with dynamic boundary conditions, Ann. Mat. Pura Appl. (4) 185 (2006), 627-648.

[47] R. Racke, S. Zheng, The Cahn-Hilliard equation with dynamical boundary conditions, Adv. Differential Equations 8 (2003), 83-110. 
[48] P. Rybka, K.-H. Hoffmann, Convergence of solutions to Cahn-Hilliard equation, Comm. Partial Differential Equations 24 (1999), 1055-1077.

[49] A. Segatti, On the hyperbolic relaxation of the Cahn-Hilliard equation in 3D: approximation and long time behaviour, Math. Models Methods Appl. Sci. 17 (2007), 411-437.

[50] V. Vergara, A conserved phase field system with memory and relaxed chemical potential, J. Math. Anal. Appl. 328 (2007), no. 2, 789-812.

[51] H. Wu, S. Zheng, Convergence to equilibrium for the Cahn-Hilliard equation with dynamic boundary conditions, J. Differential Equations 204 (2004), 511-531.

[52] R. Temam, "Infinite-Dimensional Dynamical Systems in Mechanics and Physics", Springer-Verlag, New York, 1997. 